Review

\title{
Biocidal Agents Used for Disinfection Can Enhance Antibiotic Resistance in Gram-Negative Species
}

\author{
Günter Kampf \\ University Medicine Greifswald, Institute for Hygiene and Environmental Medicine, \\ 17475 Greifswald, Germany; guenter.kampf@uni-greifswald.de
}

Received: 20 November 2018; Accepted: 11 December 2018; Published: 14 December 2018

\begin{abstract}
Biocidal agents used for disinfection are usually not suspected to enhance cross-resistance to antibiotics. The aim of this review was therefore to evaluate the effect of 13 biocidal agents at sublethal concentrations on antibiotic resistance in Gram-negative species. A medline search was performed for each biocidal agent on antibiotic tolerance, antibiotic resistance, horizontal gene transfer, and efflux pump. In cells adapted to benzalkonium chloride a new resistance was most frequently found to ampicillin (eight species), cefotaxime (six species), and sulfamethoxazole (three species), some of them with relevance for healthcare-associated infections such as Enterobacter cloacae or Escherichia coli. With chlorhexidine a new resistance was often found to ceftazidime, sulfamethoxazole and imipenem (eight species each) as well as cefotaxime and tetracycline (seven species each). Cross-resistance to antibiotics was also found with triclosan, octenidine, sodium hypochlorite, and didecyldimethylammonium chloride. No cross-resistance to antibiotics has been described after low level exposure to ethanol, propanol, peracetic acid, polyhexanide, povidone iodine, glutaraldehyde, and hydrogen peroxide. Taking into account that some biocidal agents used in disinfectants have no health benefit (e.g., in alcohol-based hand rubs) but may cause antibiotic resistance it is obvious to prefer products without them.
\end{abstract}

Keywords: biocide; cross-resistance; cross-tolerance; antibiotics; antiseptic stewardship

\section{Introduction}

Biocidal agents used for disinfection are one of many elements to limit the spread of antibiotic resistant bacteria. Most users of disinfectants would not expect that biocidal agents may cause antibiotic resistance themselves. Triclosan is such an example. It was used for decades in antimicrobial soaps in the US and considered to be safe and effective [1]. But in 201619 active ingredients including triclosan were banned by the US Food and Drug Administration for antimicrobial soaps used at home by the general population [2]. The decision was justified by associated risks including antibiotic resistance and a lack of a health benefit: "A risk must be balanced that demonstrate a direct clinical benefit (i.e., a reduction of infection) - that the product is superior to washing with non-antibacterial soap and water in reducing infection." The scientific community welcomed the decision: "We applaud this rule specifically because of the associated risks that triclosan poses to the spread of antibiotic resistance throughout the environment. This persistent chemical constantly stresses bacteria to adapt, and behavior that promotes antibiotic resistance needs to be stopped immediately when the benefits are null" [3].

Other biocidal agents used for disinfection in healthcare, veterinary medicine, food production, food handling or in the domestic setting may also have a risk of enhancing antibiotic resistance, especially during low level exposure [4]. Persistence of the biocidal agent is certainly an advantage for an adaptive response. However, there is currently a lack of awareness in the infection control community that some biocidal agents may have a larger risk for promoting antibiotic resistance than 
others. The aim of the review is therefore to summarize data on the development of antibiotic tolerance and resistance, changes of horizontal gene transfer, induction of antibiotic resistance genes, and the effect on common efflux pump genes in Gram-negative species caused by low level exposure to commonly used biocidal agents.

The following biocidal agents were reviewed: triclosan, benzalkonium chloride, hydrogen peroxide, glutaraldehyde, ethanol, chlorhexidine digluconate, sodium hypochlorite, didecyldimethylammonium chloride (DDAC), octenidine, peracetic acid, propanol, polihexanide, and povidone iodine. The medline search for "horizontal gene transfer", antibiotic, and each biocidal agent on 31 August 2018 revealed four hits for hydrogen peroxide, three hits for ethanol, three hits for chlorhexidine digluconate, two hits for triclosan, one hit for benzalkonium chloride, and 0 hits for glutaraldehyde, sodium hypochlorite, propanol, povidone iodine, peracetic acid, polihexanide, DDAC and octenidine. The medline search for "cross tolerance", antibiotic, and each biocidal agent on 19 November 2018 revealed five hits for benzalkonium chloride and hydrogen peroxide, three hits for ethanol, two hits for sodium hypochlorite and povidone iodine, one hit for chlorhexidine digluconate and polihexanide, and 0 hits for propanol, DDAC, octenidine, and peracetic acid. The medline search for "cross resistance", antibiotic and each biocidal agent on 19 November 2018 revealed 44 hits for triclosan, 36 hits for benzalkonium chloride, 32 hits for hydrogen peroxide, 23 hits for ethanol, 22 hits for povidone iodine, 19 hits for glutaraldehyde, 11 hits for sodium hypochlorite, eight hits for chlorhexidine digluconate, seven hits for peracetic acid, five hits for triclosan, two hits for propanol, polihexanide, octenidine, and glutaraldehyde, and one hit for DDAC. The medline search for "efflux pump", antibiotic and each biocidal agent on 19 November 2018 revealed 35 hits for triclosan, 31 hits for benzalkonium chloride, 10 hits for hydrogen peroxide, three hits for glutaraldehyde and ethanol, two hits for chlorhexidine digluconate, one hit for sodium hypochlorite and 0 hits for DDAC, octenidine, peracetic acid, propanol, polihexanide, and povidone iodine.

Publications were included and results were extracted from them when they provided original data on an adaptive response to the exposure of Gram-negative bacteria to sublethal concentrations of the biocidal agents described above resulting in a tolerance or resistance to antibiotics including antibiotic resistance gene changes, in a change of efflux pump activity or horizontal gene transfer. Articles were excluded when they described changes in Gram-positive species, fungi or mycobacteria. Reviews were also excluded but screened for any information within the scope of the review.

\section{Benzalkonium Chloride}

\subsection{Antibiotic Tolerance or Resistance after Low Level Biocide Exposure}

In one study it was described that exposure of Escherichia coli to low level benzalkonium chloride can increase the tolerance to benzalkonium chloride 2.6-fold and in addition also 3.3-fold to 7-fold to various antibiotics. A classification to susceptibility categories, however, was not found (Table 1).

In 11 studies an associated increase of tolerance or a new resistance to antibiotics was described for some Gram-negative species (Table 2). A new resistance was most frequently found to ampicillin (eight species), cefotaxime (six species), and sulfamethoxazole (three species). For two species a new antibiotic resistance was detected for ceftazidime, trimethoprim-sulfamethoxazol, trimethoprim, tetracycline, imipenem, chloramphenicol, amoxicillin, or amoxicillin-clavulanic acid. Only one species was resistant to nalidixic acid or ceftriaxone. Among the species some have a major relevance for healthcare-associated infections such as Enterobacter cloacae or Escherichia coli. 
Table 1. Gram-negative species with increased antibiotic tolerance after various types of low level exposure ( $<$ MIC value) to benzalkonium chloride (BAC).

\begin{tabular}{|c|c|c|c|c|c|}
\hline Species & Strain(s) & $\begin{array}{l}\text { MIC Increase } \\
\text { (BAC) }\end{array}$ & Antibiotic(s) & $\begin{array}{l}\text { MIC Increase } \\
\text { (Antibiotic) }\end{array}$ & Reference \\
\hline Escherichia coli & $\begin{array}{l}\text { ATCC } 25922 \\
\text { and } 9 \text { avian and } \\
\text { porcine strains }\end{array}$ & 2.6-fold & $\begin{array}{c}\text { Florfenicol } \\
\text { Cefotaxime } \\
\text { Chloramphenicol } \\
\text { Ceftazidime } \\
\text { Nalidixic acid } \\
\text { Ampicillin } \\
\text { Tetracycline } \\
\text { Ciprofloxacin } \\
\text { Sulfamethoxazole } \\
\text { Trimethoprim }\end{array}$ & 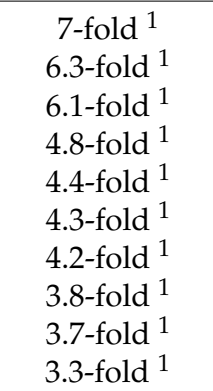 & [5] \\
\hline
\end{tabular}

${ }^{1}$ microdilution method (mg/L). 
Table 2. Gram-negative species with antibiotic resistance after various types of low level exposure ( $<$ MIC value) to BAC.

\begin{tabular}{|c|c|c|c|c|c|c|c|}
\hline Species & Strain(s) & $\begin{array}{l}\text { MIC Increase } \\
\text { (BAC) }\end{array}$ & Antibiotic(s) & Pre-Value & Post-Value & Category & Reference \\
\hline $\begin{array}{l}\text { Burkholderia } \\
\text { cepacia complex }\end{array}$ & $\begin{array}{l}\text { B. lata strain } 383 \\
\text { (4 experiments) }\end{array}$ & - & $\begin{array}{l}\text { Imipenem } \\
\text { Meropenem } \\
\text { Ciprofloxacin } \\
\text { Ceftazidime } \\
\text { Tobramycin }\end{array}$ & $\begin{array}{c}24^{1} \\
40.7^{1} \\
30^{1} \\
40.3^{1} \\
7.3^{1}\end{array}$ & $\begin{array}{c}16(1)^{1} \\
34-35.5(2)^{1} \\
12-24(2)^{1} \\
12(1)^{1} \\
0(1)^{1}\end{array}$ & $\begin{array}{l}- \\
- \\
- \\
-\end{array}$ & [6] \\
\hline Chryseobacterium spp. & $\begin{array}{l}\text { Biocide-sensitive strain } \\
\text { from organic foods }\end{array}$ & 20-fold & Ampicillin & - & $64^{1}$ & $\mathrm{R}$ & [7] \\
\hline Enterobacter cloacae & $\begin{array}{l}\text { Two biocide-sensitive } \\
\text { strains from organic foods }\end{array}$ & 12-fold-30-fold & $\begin{array}{l}\text { Cefotaxime } \\
\text { Ampicillin }\end{array}$ & - & $\begin{array}{l}128(1)^{1} \\
64(1)^{1}\end{array}$ & $\begin{array}{l}\mathrm{R} \\
\mathrm{R}\end{array}$ & [7] \\
\hline Enterobacter ludwigii & $\begin{array}{l}\text { Biocide-sensitive strain } \\
\text { from organic foods }\end{array}$ & 30-fold & Cefotaxime & - & $128^{1}$ & $\mathrm{R}$ & [7] \\
\hline Enterobacter spp. & $\begin{array}{l}\text { Six biocide-sensitive } \\
\text { strains from organic foods }\end{array}$ & 5-fold-300-fold & $\begin{array}{c}\text { Ampicillin } \\
\text { Sulfamethoxazol } \\
\text { Ceftazidime } \\
\text { Cefotaxime } \\
\text { Trimethoprim-sulfamethoxazol }\end{array}$ & $\begin{array}{l}- \\
- \\
- \\
-\end{array}$ & $\begin{array}{c}64(5)^{1} \\
1014(2)^{1} \\
64(1)^{1} \\
64(1)^{1} \\
8 / 152(1)^{1}\end{array}$ & $\begin{array}{l}\mathrm{R} \\
\mathrm{R} \\
\mathrm{R} \\
\mathrm{R} \\
\mathrm{R}\end{array}$ & [7] \\
\hline Escherichia coli & ATCC 11775 & 6-fold & $\begin{array}{l}\text { Ampicillin } \\
\text { Chloramphenicol } \\
\text { Erythromycin } \\
\text { Gentamicin } \\
\text { Kanamycin } \\
\text { Nalidixic acid } \\
\text { Norfloxacin } \\
\text { Penicillin } \\
\text { Tetracycline }\end{array}$ & $\begin{array}{c}10^{1} \\
10^{1} \\
140^{1} \\
2^{1} \\
8^{1} \\
8^{1} \\
0.15^{1} \\
250^{1} \\
4^{1}\end{array}$ & $\begin{array}{c}50^{1} \\
240^{1} \\
180^{1} \\
4^{1} \\
16^{1} \\
30^{1} \\
0.4^{1} \\
400^{1} \\
16^{1}\end{array}$ & $\begin{array}{l}- \\
- \\
- \\
- \\
- \\
- \\
- \\
-\end{array}$ & [8] \\
\hline Escherichia coli & DSM 682 & 6-fold & $\begin{array}{l}\text { Ampicillin } \\
\text { Chloramphenicol } \\
\text { Erythromycin } \\
\text { Gentamicin } \\
\text { Kanamycin } \\
\text { Nalidixic acid } \\
\text { Norfloxacin } \\
\text { Penicillin } \\
\text { Tetracycline }\end{array}$ & $\begin{array}{c}5^{1} \\
5^{1} \\
100^{1} \\
2^{1} \\
10^{1} \\
4^{1} \\
0.1^{1} \\
100^{1} \\
4^{1}\end{array}$ & $\begin{array}{c}20{ }^{1} \\
60^{1} \\
160^{1} \\
4^{1} \\
10^{1} \\
30^{1} \\
0.15^{1} \\
200^{1} \\
6^{1}\end{array}$ & $\begin{array}{c}- \\
- \\
- \\
- \\
\text { n.a. } \\
- \\
- \\
- \\
-\end{array}$ & [8] \\
\hline Escherichia coli & ATCC 47076 & 6-fold-7-fold & $\begin{array}{l}\text { Chloramphenicol } \\
\text { Florfenicol } \\
\text { Ciprofloxacin } \\
\text { Nalidixic acid } \\
\text { Ampicillin } \\
\text { Cefotaxime }\end{array}$ & $\begin{array}{c}8^{1} \\
8^{1} \\
0.06^{1} \\
8^{1} \\
4^{1} \\
0.06^{1}\end{array}$ & $\begin{array}{c}8-128^{1} \\
16-64^{1} \\
0.25^{1} \\
32-64^{1} \\
4-8^{1} \\
0.12-0.5^{1}\end{array}$ & $\begin{array}{l}- \\
- \\
- \\
- \\
-\end{array}$ & [9] \\
\hline
\end{tabular}


Table 2. Cont

\begin{tabular}{|c|c|c|c|c|c|c|c|}
\hline Species & Strain(s) & $\begin{array}{l}\text { MIC Increase } \\
\text { (BAC) }\end{array}$ & Antibiotic(s) & Pre-Value & Post-Value & Category & Reference \\
\hline Escherichia coli & NCTC 12900 strain O157 & Approx. 100-fold & $\begin{array}{c}\text { Amoxicillin-clavulanic acid } \\
\text { Amoxicillin } \\
\text { Chloramphenicol } \\
\text { Ciprofloxacin } \\
\text { Clindamycin } \\
\text { Colistin sulfate } \\
\text { Erythromycin } \\
\text { Fusidic acid } \\
\text { Gentamicin } \\
\text { Imipenem } \\
\text { Rifampicin } \\
\text { Tetracycline } \\
\text { Trimethoprim } \\
\text { Vancomycin }\end{array}$ & $\begin{array}{c}12^{2} \\
12^{2} \\
19^{2} \\
14^{2} \\
0^{2} \\
10^{2} \\
4^{2} \\
0^{2} \\
13^{2} \\
15^{2} \\
5^{2} \\
10^{2} \\
14^{2} \\
0^{2}\end{array}$ & $\begin{array}{c}0^{2} \\
0^{2} \\
0^{2} \\
14^{2} \\
0^{2} \\
104^{2} \\
0^{2} \\
13^{2} \\
10^{2} \\
5^{2} \\
4^{2} \\
0^{2} \\
0^{2}\end{array}$ & $\begin{array}{l}\mathrm{R} \\
\mathrm{R} \\
\mathrm{R} \\
\text { n. a. } \\
\text { n. a. } \\
\text { n. a. } \\
\text { n. a. } \\
\text { n. a. } \\
\text { n. a. } \\
\mathrm{R} \\
\text { n. a. } \\
\mathrm{R} \\
\mathrm{R} \\
\text { n. a. }\end{array}$ & [10] \\
\hline $\begin{array}{l}\text { Escherichia coli and } \\
\text { Salmonella spp. } \\
\text { (non-typhoidal) }\end{array}$ & $\begin{array}{c}12 \text { pan-susceptible strains } \\
\text { (6 per species) }\end{array}$ & $24 \%^{4}$ & $\begin{array}{c}\text { Tetracycline } \\
\text { Ciprofloxacin } \\
\text { Chloramphenicol } \\
\text { Trimethoprim-Sulfamethoxazol } \\
\text { Ampicillin } \\
\text { Gentamicin }\end{array}$ & $\begin{array}{c}2.4^{3,4} \\
0.03^{3,4} \\
6.5^{3,4} \\
0.09^{3,4} \\
18.6^{2,4} \\
1.1^{3,4}\end{array}$ & $\begin{array}{c}23.3^{3,4} \\
0.11^{3,4} \\
13.7^{3,4} \\
0.14^{3,4} \\
12.0^{2,4} \\
1.3^{3,4}\end{array}$ & $\begin{array}{l}\mathrm{R}(5) \\
\mathrm{S} \\
\mathrm{I}(6) \\
\mathrm{S} \\
\mathrm{R}(6) \\
\mathrm{S}\end{array}$ & [11] \\
\hline Klebsiella oxytoca & $\begin{array}{l}\text { Biocide-sensitive strain } \\
\text { from organic foods }\end{array}$ & 3 -fold & $\begin{array}{c}\text { Ampicillin } \\
\text { Cefotaxime } \\
\text { Ciprofloxacin } \\
\text { Imipenem } \\
\text { Ceftazidime } \\
\text { Tetracycline } \\
\text { Trimethoprim-Sulfamethoxazol } \\
\text { Sulfamethoxazol } \\
\text { Nalidixic acid }\end{array}$ & $\begin{array}{r}\text { No cl } \\
\quad(a l\end{array}$ & $\begin{array}{l}\text { ance }{ }^{1} \\
\text { tics) }\end{array}$ & n. a. & [7] \\
\hline Klebsiella spp. & $\begin{array}{l}\text { Biocide-sensitive strain } \\
\text { from organic foods }\end{array}$ & 36-fold & Ampicillin & - & $64^{1}$ & $\mathrm{R}$ & [7] \\
\hline Pantoea agglomerans & $\begin{array}{l}\text { Four biocide-sensitive } \\
\text { strains from organic foods }\end{array}$ & 20-fold-70-fold & $\begin{array}{l}\text { Ampicillin } \\
\text { Ceftazidime } \\
\text { Cefotaxime }\end{array}$ & $\begin{array}{l}- \\
- \\
-\end{array}$ & $\begin{array}{c}64(4)^{1} \\
32-64(2)^{1} \\
128(1)^{1} \\
\end{array}$ & $\begin{array}{l}\mathrm{R} \\
\mathrm{R} \\
\mathrm{R}\end{array}$ & [7] \\
\hline Pantoea ananatis & $\begin{array}{l}\text { Biocide-sensitive strain } \\
\text { from organic foods }\end{array}$ & 25-fold & $\begin{array}{c}\text { Ampicillin } \\
\text { Cefotaxime } \\
\text { Sulfamethoxazol }\end{array}$ & $\begin{array}{l}- \\
- \\
-\end{array}$ & $\begin{array}{c}64^{1} \\
64^{1} \\
1024^{1} \\
\end{array}$ & $\begin{array}{l}\mathrm{R} \\
\mathrm{R} \\
\mathrm{R}\end{array}$ & [7] \\
\hline Pantoea spp. & $\begin{array}{l}\text { Three biocide-sensitive } \\
\text { strains from organic foods }\end{array}$ & 100-fold-500-fold & $\begin{array}{c}\text { Ampicillin } \\
\text { Cefotaxime } \\
\text { Sulfamethoxazol }\end{array}$ & $\begin{array}{l}- \\
- \\
-\end{array}$ & $\begin{array}{c}64(1)^{1} \\
128(1)^{1} \\
1024(1)^{1}\end{array}$ & $\begin{array}{l}\mathrm{R} \\
\mathrm{R} \\
\mathrm{R}\end{array}$ & [7] \\
\hline
\end{tabular}


Table 2. Cont

\begin{tabular}{|c|c|c|c|c|c|c|c|}
\hline Species & Strain(s) & $\begin{array}{l}\text { MIC Increase } \\
\text { (BAC) }\end{array}$ & Antibiotic(s) & Pre-Value & Post-Value & Category & Reference \\
\hline $\begin{array}{l}\text { Pseudomonas } \\
\text { aeruginosa }\end{array}$ & $\begin{array}{l}22 \text { isolates from biofilm } \\
\text { samples in dairy }\end{array}$ & $\leq 2.2$-fold & Ciprofloxacin & $0.25-32^{1}$ & $3.5-55^{1,5}$ & - & [12] \\
\hline $\begin{array}{l}\text { Pseudomonas } \\
\text { aeruginosa }\end{array}$ & Strain NCIMB 10421 & 12-fold & $\begin{array}{l}\text { Amikacin } \\
\text { Ceftazidime } \\
\text { Ciprofloxacin } \\
\text { Gentamycin } \\
\text { Imipenem } \\
\text { Ticarcillin }\end{array}$ & $\begin{array}{c}3.5^{3} \\
2^{3} \\
0.125^{3} \\
2.5^{3} \\
2^{3} \\
0.875^{3}\end{array}$ & $\begin{array}{c}1.75^{3} \\
0.44^{3} \\
0.047^{3} \\
0.75^{3} \\
0.5^{3} \\
0.285^{3}\end{array}$ & $\begin{array}{l}\text { n. a. } \\
\text { n. a. } \\
\text { n. a. } \\
\text { n. a. } \\
\text { n. a. } \\
\text { n. a. }\end{array}$ & [13] \\
\hline $\begin{array}{l}\text { Pseudomonas } \\
\text { aeruginosa }\end{array}$ & Strain NCIMB 10421 & $>12$-fold & $\begin{array}{c}\text { Ciprofloxacin } \\
\text { Tobramycin } \\
\text { Minocycline } \\
\text { Aztreonam } \\
\text { Polymyxin B } \\
\text { Amikacin } \\
\text { Gentamicin } \\
\text { Vancomycin } \\
\text { Imipenem }\end{array}$ & $\begin{array}{c}0.125^{3} \\
1.5^{3} \\
>128^{3} \\
3^{3} \\
4^{3} \\
8^{3} \\
4^{3} \\
>128^{3} \\
2^{3}\end{array}$ & $\begin{array}{c}32^{3} \\
1.0^{3} \\
16^{3} \\
3^{3} \\
2^{3} \\
6^{3} \\
6^{3} \\
>128^{3} \\
2^{3}\end{array}$ & $\begin{array}{l}- \\
- \\
- \\
- \\
- \\
- \\
- \\
-\end{array}$ & [14] \\
\hline $\begin{array}{c}\text { Pseudomonas } \\
\text { aeruginosa }\end{array}$ & $\begin{array}{l}\text { Isolate from } \\
\text { river sediment }\end{array}$ & 4 -fold & Polymyxin B & $0.2-0.4^{1}$ & $0.8-1.6^{1}$ & - & [15] \\
\hline Salmonella Enteritidis & Clinical isolate & Approx. 200-fold & Various antibiotics & \multicolumn{2}{|c|}{ No cross-resistance ${ }^{2}$} & n.a. & [10] \\
\hline Salmonella Hvittingfoss & Strain S41 & 4-fold & $\begin{array}{c}\text { Ampicillin } \\
\text { Amoxicillin-clavulanic acid } \\
\text { Piperacillin } \\
\text { Cephalexin } \\
\text { Cefpodoxime } \\
\text { Ceftiofur } \\
\text { Ceftriaxone } \\
\text { Tetracycline } \\
\text { Ciprofloxacin } \\
\text { Chloramphenicol } \\
\text { Cefoxitin } \\
\text { Nalidixic acid }\end{array}$ & $\begin{aligned} &<2^{6} \\
&<2 \\
&<4^{6} \\
&<4^{6} \\
&<0.25^{6} \\
&<1^{6} \\
&<0.25^{6} \\
&<1^{6} \\
& 0.06^{6} \\
& 4^{6} \\
& 8^{6} \\
& 4^{6}\end{aligned}$ & $\begin{array}{c}16^{6} \\
4^{6} \\
64^{6} \\
16^{6} \\
2^{6} \\
>8^{6} \\
2^{6} \\
8^{6} \\
0.5^{6} \\
16^{6} \\
>32^{6} \\
32^{6}\end{array}$ & $\begin{array}{l}\text { I } \\
- \\
\text { I } \\
\text { I } \\
\text { I } \\
\text { I } \\
\text { R } \\
\text { I } \\
\text { I } \\
\text { I } \\
- \\
\text { R }\end{array}$ & [16] \\
\hline
\end{tabular}


Table 2. Cont

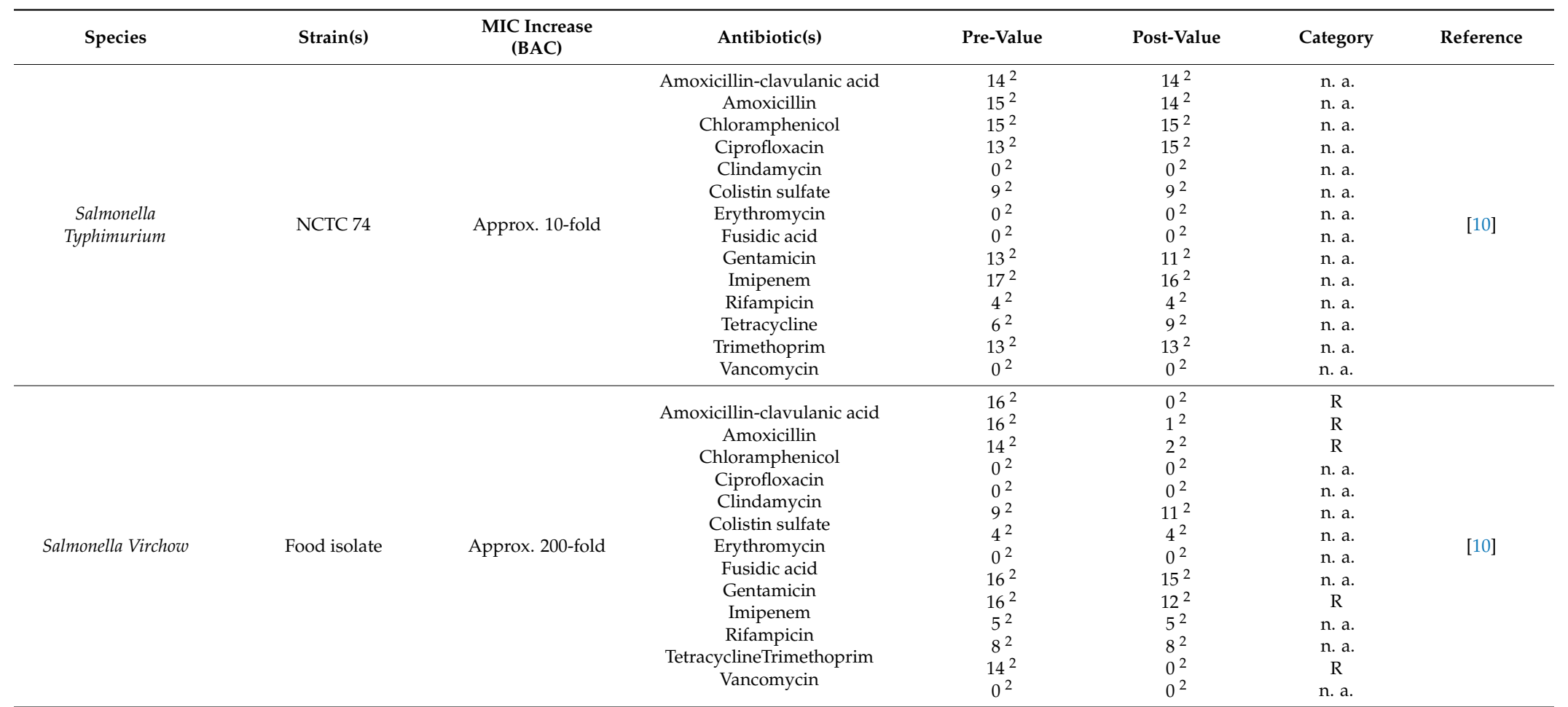

${ }^{1}$ microdilution method $(\mathrm{mg} / \mathrm{L}) ;{ }^{2}$ disc diffusion test $(\mathrm{mm}) ;{ }^{3}$ Etest $(\mathrm{mg} / \mathrm{L}) ;{ }^{4}$ mean; ${ }^{5}$ no conclusive cross-resistance; ${ }^{6}$ NARMS plates; “-" $=$ no information; $\mathrm{R}=$ resistant; $\mathrm{I}=$ intermediate susceptible; $\mathrm{S}=$ susceptible; $\mathrm{n}$. a. = not applicable; () = number of strains, isolates or experiments. 


\subsection{Effect on Antibiotic Resistance Genes}

Benzalkonium chloride has been described to co-select for other antimicrobial resistance genes [17].

\subsection{Increase of Horizontal Gene Transfer}

The general possibility of horizontal gene transfer for the spread of antibiotic and biocide resistance has been described already in 2001 [18]. In two of 179 Escherichia coli isolates from retail food qacH-associated integrons associated with tolerance to benzalkonium chloride located on $100 \mathrm{~kb}$ plasmids could be transferred to an E. coli recipient, indicating the co-existence and co-dissemination of disinfectant and antimicrobial resistance genes among bacterial species [19].

\subsection{Induction of Common Efflux Pumps}

In Pseudomonas aeruginosa, benzalkonium chloride can induce the MexCD-OprJ multidrug efflux pump [20].

\subsection{Additional Findings}

Some other studies demonstrate a correlation between tolerance to benzalkonium chloride and resistance to various antibiotics. In 153 Escherichia coli blood culture isolates, for example, a higher MIC of benzalkonium chloride was associated with a decreased susceptibility to cotrimoxazole [21]. In 52 Pseudomonas spp. from meat chain production, a correlation between resistance to benzalkonium chloride and ampicillin, amoxicillin, erythromycin, and trimethoprim was found [22]. Repeated in vitro exposure of Salmonella Typhimurium cells to quaternary ammonium compounds selects for a higher tolerance to chloramphenicol, tetracycline, ampicillin, and acriflavine which is explained by an overexpression of the AcrAB efflux pump [23]. Few other studies do not describe such a correlation. No correlation between multiple antibiotic-resistant bacteria and a tolerance to benzalkonium chloride was found in 122 isolates of Salmonella spp. from poultry and swine [24]. In analogy, no association between resistance to multiple antibiotic and quaternary ammonium compounds was found in 103 Gram-negative clinical isolates [25]. One of the reasons for a cross-resistance with benzalkonium chloride is a multidrug efflux protein MdtM which was detected in E. coli. It belongs to the large and ubiquitous major facilitator superfamily (MFS). Benzalkonium chloride, didecyldimethylammonium chloride and some antibiotics are among the substrates transported by MdtM [26]. It was also shown with $E$. coli that many redundant multidrug resistance transporters also enhance biofilm formation and drug tolerance including benzalkonium chloride [27]. Efflux pumps also explain resistance to benzalkonium chloride in P. aeruginosa [12]. In P. fluorescens high level resistance to benzalkonium chloride was also explained by an efflux system which excretes only specific cationic disinfectants belonging to the group of quaternary ammonium compounds [28]. Over-expression of efflux pumps AcrAB or AcrEF was detected in benzalkonium chloride-resistant mutants of S. Typhimurium [29].

\section{Chlorhexidine Digluconate}

\subsection{Antibiotic Tolerance or Resistance after Low Level Biocide Exposure}

Six studies indicate that low level chlorhexidine exposure quite often results in an antibiotic resistance, so far mainly described in biocide-sensitive strains from organic foods (Table 3). A new resistance was most frequently found to ceftazidime, sulfamethoxazole, and imipenem (eight species each) as well as cefotaxime and tetracycline (seven species each). For two species a new antibiotic resistance was detected for ampicillin. Only one species was finally resistant to nalidixic acid, colistin, or tobramycin. Among the species some have also relevance for healthcare such as Enterobacter cloacae, Escherichia coli, or Klebsiella pneumoniae. 
Table 3. Gram-negative species with antibiotic resistance after various types of low level exposure (<MIC value) to chlorhexidine digluconate (CHG).

\begin{tabular}{|c|c|c|c|c|c|c|c|}
\hline Species & Strain(s) & $\begin{array}{l}\text { MIC Increase } \\
\text { (CHG) }\end{array}$ & Antibiotic(s) & Pre-Value & Post-Value & Category & Reference \\
\hline Bacteroides fragilis & ATCC 25285 & - & $\begin{array}{l}\text { Ampicillin } \\
\text { Cefoxitin } \\
\text { Cefoperazone } \\
\text { Chloramphenicol } \\
\text { Metronidazole } \\
\text { Norfloxacin } \\
\text { Tetracycline }\end{array}$ & $\begin{array}{c}46^{1} \\
7^{1} \\
52^{1} \\
2^{1} \\
0.6^{1} \\
0.6^{1} \\
0.6^{1}\end{array}$ & $\begin{array}{c}77^{1} \\
13^{1} \\
126^{1} \\
2^{1} \\
0.9^{1} \\
0.9^{1} \\
2^{1}\end{array}$ & $\begin{array}{l}- \\
- \\
- \\
- \\
- \\
-\end{array}$ & [30] \\
\hline $\begin{array}{l}\text { Burkholderia cepacia } \\
\text { complex }\end{array}$ & B. lata strain 383 & - & $\begin{array}{l}\text { Imipenem } \\
\text { Meropenem } \\
\text { Ciprofloxacin } \\
\text { Ceftazidime } \\
\text { Tobramycin }\end{array}$ & $\begin{array}{c}24^{2} \\
40.7^{2} \\
30^{2} \\
40.3^{2} \\
7.3^{2} \\
\end{array}$ & $\begin{array}{c}15-21(2)^{2} \\
33(1)^{2} \\
11-20(2)^{2} \\
30-33(2)^{2} \\
-\end{array}$ & $\begin{array}{l}- \\
- \\
- \\
-\end{array}$ & [6] \\
\hline Chrysobacterium spp. & $\begin{array}{c}2 \text { biocide-sensitive } \\
\text { strains from } \\
\text { organic foods }\end{array}$ & 5-fold-6-fold & $\begin{array}{c}\text { Ampicillin } \\
\text { Cefotaxime } \\
\text { Ceftazidime } \\
\text { Sulfamethoxazol } \\
\text { Tetracycline }\end{array}$ & $\begin{array}{l}- \\
- \\
- \\
-\end{array}$ & $\begin{array}{c}64(1)^{2} \\
128(2)^{2} \\
64(2)^{2} \\
1024(1)^{2} \\
16(1)^{2}\end{array}$ & $\begin{array}{l}\mathrm{R} \\
\mathrm{R} \\
\mathrm{R} \\
\mathrm{R} \\
\mathrm{R}\end{array}$ & [31] \\
\hline Enterobacter cloacae & $\begin{array}{c}2 \text { biocide-sensitive } \\
\text { strains from } \\
\text { organic foods }\end{array}$ & 10-fold-16-fold & $\begin{array}{l}\text { Cefotaxime } \\
\text { Ceftazidime } \\
\text { Imipenem } \\
\text { Sulfamethoxazol } \\
\text { Tetracycline }\end{array}$ & $\begin{array}{l}- \\
- \\
- \\
-\end{array}$ & $\begin{array}{c}64(1)^{2} \\
64(2)^{2} \\
16(2)^{2} \\
1024(2)^{2} \\
32(1)^{2}\end{array}$ & $\begin{array}{l}\mathrm{R} \\
\mathrm{R} \\
\mathrm{R} \\
\mathrm{R} \\
\mathrm{R}\end{array}$ & [31] \\
\hline Enterobacter ludwigii & $\begin{array}{c}2 \text { biocide-sensitive } \\
\text { strains from } \\
\text { organic foods }\end{array}$ & 6-fold-8-fold & $\begin{array}{c}\text { Ceftazidime } \\
\text { Imipenem } \\
\text { Sulfamethoxazol }\end{array}$ & $\begin{array}{l}- \\
- \\
-\end{array}$ & $\begin{array}{c}64(2)^{2} \\
16(2)^{2} \\
1024(2)^{2} \\
\end{array}$ & $\begin{array}{l}\mathrm{R} \\
\mathrm{R} \\
\mathrm{R}\end{array}$ & [31] \\
\hline Enterobacter spp. & $\begin{array}{l}6 \text { biocide-sensitive } \\
\text { strains from } \\
\text { organic foods }\end{array}$ & 4-fold-10-fold & $\begin{array}{l}\text { Cefotaxime } \\
\text { Ceftazidime } \\
\text { Imipenem } \\
\text { Sulfamethoxazol }\end{array}$ & $\begin{array}{l}- \\
- \\
- \\
- \\
-\end{array}$ & $\begin{array}{c}64(1)^{2} \\
128(1)^{2} \\
64(3)^{2} \\
16(3)^{2} \\
1024(2)^{2} \\
\end{array}$ & $\begin{array}{l}\mathrm{R} \\
\mathrm{R} \\
\mathrm{R} \\
\mathrm{R} \\
\mathrm{R}\end{array}$ & [31] \\
\hline Escherichia coli & NCIMB 8545 & $\leq 6$-fold & Tobramycin & - & -2 & $\mathrm{R}^{3}$ & [32] \\
\hline
\end{tabular}


Table 3. Cont.

\begin{tabular}{|c|c|c|c|c|c|c|c|}
\hline Species & Strain(s) & $\begin{array}{l}\text { MIC Increase } \\
\text { (CHG) }\end{array}$ & Antibiotic(s) & Pre-Value & Post-Value & Category & Reference \\
\hline Escherichia coli & $\begin{array}{l}\text { NCTC } 12900 \\
\text { strain O157 }\end{array}$ & Approx. 50-fold & Various antibiotics & \multicolumn{2}{|c|}{ No cross-resistance 4} & n.a. & [10] \\
\hline Klebsiella oxytoca & $\begin{array}{c}2 \text { biocide-sensitive } \\
\text { strains from } \\
\text { organic foods }\end{array}$ & 2-fold-8-fold & Various antibiotics & \multicolumn{2}{|c|}{ No cross-resistance ${ }^{2}$} & n.a. & [31] \\
\hline Klebsiella pneumoniae & $\begin{array}{l}6 \text { clinical strains } \\
\text { with a variety of } \\
\text { antibiotic } \\
\text { resistance markers }\end{array}$ & 4-fold-16-fold & $\begin{array}{l}\text { Azithromycin } \\
\text { Cefepime } \\
\text { Colistin } \\
\text { Teicoplanin }\end{array}$ & $\begin{array}{c}8-64(6) \\
0.06-0.125(1) \\
\geq 64(5) \\
2-4(6) \\
>64(6)\end{array}$ & $\begin{array}{c}8-64(6)^{2} \\
0.06-0.5(2)^{2} \\
\geq 64(4)^{2} \\
>64(5)^{2} \\
>64(6)^{2}\end{array}$ & $\begin{array}{l}\text { n.a. } \\
\text { n.a. } \\
\text { n.a. } \\
\text { R } \\
\text { n.a. }\end{array}$ & [33] \\
\hline Klebsiella spp. & $\begin{array}{l}\text { Biocide-sensitive } \\
\text { strain from } \\
\text { organic foods }\end{array}$ & 2-fold & $\begin{array}{l}\text { Ceftazidime } \\
\text { Imipenem }\end{array}$ & - & $\begin{array}{l}64^{2} \\
16^{2}\end{array}$ & $\begin{array}{l}\mathrm{R} \\
\mathrm{R}\end{array}$ & [31] \\
\hline Pantoea agglomerans & $\begin{array}{c}5 \text { biocide-sensitive } \\
\text { strains from } \\
\text { organic foods }\end{array}$ & 5-fold-10-fold & $\begin{array}{l}\text { Cefotaxime } \\
\text { Ceftazidime } \\
\text { Imipenem } \\
\text { Sulfamethoxazol } \\
\text { Tetracycline }\end{array}$ & $\begin{array}{l}- \\
- \\
- \\
-\end{array}$ & $\begin{array}{c}64-128(3)^{2} \\
64(3)^{2} \\
16(1)^{2} \\
1024(2)^{2} \\
16-32(2)^{2}\end{array}$ & $\begin{array}{l}\mathrm{R} \\
\mathrm{R} \\
\mathrm{R} \\
\mathrm{R} \\
\mathrm{R}\end{array}$ & [31] \\
\hline Pantoea ananatis & $\begin{array}{c}2 \text { biocide-sensitive } \\
\text { strains from } \\
\text { organic foods }\end{array}$ & 10-fold-50-fold & $\begin{array}{l}\text { Cefotaxime } \\
\text { Ceftazidime } \\
\text { Imipenem } \\
\text { Sulfamethoxazol } \\
\text { Tetracycline }\end{array}$ & $\begin{array}{l}- \\
- \\
- \\
-\end{array}$ & $\begin{array}{c}64-128(2)^{2} \\
64(1)^{2} \\
16(1)^{2} \\
1024(1)^{2} \\
16(1)^{2}\end{array}$ & $\begin{array}{l}\mathrm{R} \\
\mathrm{R} \\
\mathrm{R} \\
\mathrm{R} \\
\mathrm{R}\end{array}$ & [31] \\
\hline Pantoea spp. & $\begin{array}{c}3 \text { biocide-sensitive } \\
\text { strains from } \\
\text { organic foods }\end{array}$ & 5-fold-16-fold & $\begin{array}{l}\text { Ampicillin } \\
\text { Cefotaxime } \\
\text { Ceftazidime } \\
\text { Imipenem } \\
\text { Sulfamethoxazol } \\
\text { Tetracycline }\end{array}$ & $\begin{array}{l}- \\
- \\
- \\
- \\
-\end{array}$ & $\begin{array}{c}32(1)^{2} \\
128(1)^{2} \\
64(1)^{2} \\
16(1)^{2} \\
1024(1)^{2} \\
16-32(2)^{2}\end{array}$ & $\begin{array}{l}\mathrm{R} \\
\mathrm{R} \\
\mathrm{R} \\
\mathrm{R} \\
\mathrm{R} \\
\mathrm{R}\end{array}$ & [31] \\
\hline Salmonella Virchow & Food isolate & Approx. 10-fold & Various antibiotics & No cro & stance ${ }^{4}$ & n.a. & [10] \\
\hline
\end{tabular}


Table 3. Cont.

\begin{tabular}{|c|c|c|c|c|c|c|c|}
\hline Species & Strain(s) & $\begin{array}{l}\text { MIC Increase } \\
\text { (CHG) }\end{array}$ & Antibiotic(s) & Pre-Value & Post-Value & Category & Reference \\
\hline Salmonella spp. & $\begin{array}{c}3 \text { biocide-sensitive } \\
\text { strains from } \\
\text { organic foods }\end{array}$ & 5-fold-10-fold & $\begin{array}{c}\text { Cefotaxime } \\
\text { Imipenem } \\
\text { Nalidixic acid } \\
\text { Sulfamethoxazol } \\
\text { Tetracycline }\end{array}$ & $\begin{array}{l}- \\
- \\
-\end{array}$ & $\begin{array}{c}128(2)^{2} \\
16(2)^{2} \\
64(2)^{2} \\
1024(1)^{2} \\
32(1)^{2}\end{array}$ & $\begin{array}{l}\mathrm{R} \\
\mathrm{R} \\
\mathrm{R} \\
\mathrm{R} \\
\mathrm{R}\end{array}$ & [31] \\
\hline Salmonella spp. & $\begin{array}{l}6 \text { strains with } \\
\text { higher MICs to } \\
\text { biocidal products }\end{array}$ & $\begin{array}{l}\text { 50-fold-200-fold } \\
\text { (2 strains) }\end{array}$ & $\begin{array}{c}\text { Tetracycline } \\
\text { Chloramphenicol } \\
\text { Nalidixic acid }\end{array}$ & $\begin{array}{c}<1^{4} \\
4^{4} \\
4^{4}\end{array}$ & $\begin{array}{l}>16(1)^{5} \\
8(1)^{5} \\
16(1)^{5}\end{array}$ & $\begin{array}{l}\mathrm{R} \\
\mathrm{I} \\
\mathrm{I}\end{array}$ & [16] \\
\hline
\end{tabular}

${ }^{1}$ spiral gradient endpoint method $(\mathrm{mg} / \mathrm{L}) ;{ }^{2}$ microdilution method $(\mathrm{mg} / \mathrm{L}) ;{ }^{3}$ unstable; ${ }^{4}$ disc diffusion test $(\mathrm{mm}) ;{ }^{5} \mathrm{NARMS}$ plates $(\mathrm{mg} / \mathrm{L}) ;-$ no information; $\mathrm{R}=$ resistant; $\mathrm{I}=$ intermediate susceptible; $\mathrm{S}=$ susceptible; () number of strains or isolates. 


\subsection{Increase of Horizontal Gene Transfer}

Horizontal transfer of mobile antibiotic resistance elements by conjugation could be significantly increased by low level exposure to chlorhexidine digluconate $(24.4 \mu \mathrm{g} / \mathrm{L})$ to a recipient Escherichia coli strain [34]. In addition, an additional sh-fabI allele was detected in clinical isolates of Staphylococcus aureus derived from Staphylococcus haemolyticus suggesting a high potential of its horizontal gene transfer [35].

\subsection{Induction of Common Efflux Pumps}

Chlorhexidine was able to induce the expression of 6 efflux pump genes (bmeB1, bmeB3, bmeB4, bmeB7, bfrA1 and bfrA2) in Bacteroides fragilis ATCC 25285 exposed for $12 \mathrm{~h}$ to $0.06 \%$ chlorhexidine [30]. It can also induce the MexCD-OprJ multidrug efflux pump in Pseudomonas aeruginosa $[20,36]$.

\subsection{Additional Findings}

A similar overall result was found for low level chlorhexidine exposure. Some additional studies demonstrate a correlation between tolerance to chlorhexidine and resistance to various antibiotics. A positive correlation between resistance to some biocidal agents (cetrimide, chlorhexidine, hexachlorophene) and to antibiotics was described in 1991 for Serratia marcescens and Alcaligenes spp. [37]. In 49 Acinetobacter baumannii strains with a reduced susceptibility to chlorhexidine a co-resistance to carbapenem, aminoglycoside, tetracyclin, and ciprofloxacin was found [38]. In Bacteroides fragilis multiple antibiotic resistance was induced by a 2.7-6.0-fold increase of 6 efflux pumps [30]. In an Escherichia coli strain an unstable resistance to tobramycin was detected after low level exposure to chlorhexidine for up to $24 \mathrm{~h}$ [32]. In Trinidad 11 of 120 chlorhexidine solutions were found to be contaminated with Pseudomonas spp., with resistance rates to ciprofloxacin of $58.3 \%$, to norfloxacin of $50.0 \%$, to tobramycin of $45.8 \%$, and to gentamicin with $41.7 \%$ [39]. In a chlorhexidine-resistant Pseudomonas stutzeri isolate a cross-resistance to polymyxin and gentamicin was found [40]. A study with six other Pseudomonas stutzeri strains revealed a cross-resistance to ampicillin in five strains, to polymyxin in four strains, to erythromycin in three strains, and to nalidixic acid and gentamicin in two strains after low level exposure to chlorhexidine diacetate for six weeks [41]. Some authors found no cross-resistance between chlorhexidine and antibiotics. For example, no correlation was found between the susceptibility to chlorhexidine and 10 different antibiotics among 101 genetically distinct isolates of the B. cepacia complex [42]. No cross-resistance was found between chlorhexidine and five antibiotics in 130 Salmonella spp. from two turkey farms [43]. And no correlation between resistance to chlorhexidine and 16 different antibiotics was found in 52 Pseudomonas spp. from meat chain production [22]. A possible cross-resistance between chlorhexidine and antibiotics is discussed controversially [44,45]. As an example, the widespread use of chlorhexidine has not yet resulted in a clinically relevant resistance to antibiotics $[46,47]$ even though the development of resistance to these agents is regarded as realistic [48].

\section{Triclosan}

\subsection{Antibiotic Tolerance or Resistance after Low Level Biocide Exposure}

Five studies indicate that low-level triclosan exposure may cause antibiotic resistance, so far also mainly described in biocide-sensitive strains from organic foods (Table 4). A new resistance was most frequently found to sulfamethoxazole (five species), ampicillin or cefotaxime (four species each) and ceftazidime, trimethoprim or chloramphenicol (three species). For two species a new antibiotic resistance was detected for amoxicillin-clavulanic acid, trimethoprim-sulfamethoxazol, amoxicillin, nalidixic acid, tetracycline, or imipenem. Only one species was resistant to erythromycin, ceftiofur, or cefoxitin. One of the species has a major relevance for healthcare-associated infections (Escherichia coli). The effect in Escherichia coli is partly explained by changes in bacterial membrane properties and enhancing the efflux system [49]. 
Table 4. Gram-negative species with antibiotic resistance after various types of low level exposure ( $<$ MIC value) to triclosan (TRI).

\begin{tabular}{|c|c|c|c|c|c|c|c|}
\hline Species & Strain(s) & MIC Increase (TRI) & Antibiotic(s) & Pre-Value & Post-Value & Category & Reference \\
\hline \multirow{2}{*}{ Actinomyces naeslundii } & \multirow{2}{*}{ Strain WVU627 } & \multirow{2}{*}{ 4.9-fold } & Metronidazole & $125^{1}$ & $125^{1}$ & - & \multirow{2}{*}{ [50] } \\
\hline & & & Tetracycline & $5.2^{1}$ & $7.8^{1}$ & - & \\
\hline \multirow{4}{*}{ Enterobacter spp. } & \multirow{4}{*}{$\begin{array}{c}5 \text { biocide-sensitive } \\
\text { strains from } \\
\text { organic foods }\end{array}$} & \multirow{4}{*}{ 2-fold-15-fold } & Ampicillin & - & $64(2)^{1}$ & $\mathrm{R}$ & \multirow{4}{*}{ [51] } \\
\hline & & & Cefotaxime & - & $128(1)^{1}$ & $\mathrm{R}$ & \\
\hline & & & Ceftazidime & - & $64(2)^{1}$ & $\mathrm{R}$ & \\
\hline & & & Sulfamethoxazol & - & $1024(2)^{1}$ & $\mathrm{R}$ & \\
\hline \multirow{2}{*}{ Escherichia coli } & \multirow{2}{*}{ ATCC 8729} & \multirow{2}{*}{ 391-fold } & Metronidazole & $250^{1}$ & $125^{1}$ & - & \multirow[b]{2}{*}{ [50] } \\
\hline & & & Tetracycline & $15.6^{1}$ & $10.4^{1}$ & - & \\
\hline \multirow{14}{*}{ Escherichia coli } & \multirow{14}{*}{$\begin{array}{l}\text { NCTC } 12900 \\
\text { strain O157 }\end{array}$} & \multirow{14}{*}{$\begin{array}{c}\text { 16-fold (P1)8192-fold } \\
\text { (P2) }\end{array}$} & Amoxicillin-clavulanic acid & $11^{2}$ & $0^{2}$ & $\mathrm{R}$ & \multirow{14}{*}{ [10] } \\
\hline & & & Amoxicillin & $13^{2}$ & $0^{2}$ & $\mathrm{R}$ & \\
\hline & & & Chloramphenicol & $13^{2}$ & $5^{2}$ & $\mathrm{R}$ & \\
\hline & & & Ciprofloxacin & $14^{2}$ & $14^{2}$ & n. a. & \\
\hline & & & Clindamycin & $0^{2}$ & $0^{2}$ & n. a. & \\
\hline & & & Colistin sulfate & $9^{2}$ & $10^{2}$ & n. a. & \\
\hline & & & Erythromycin & $7^{2}$ & $0^{2}$ & $\mathrm{R}$ & \\
\hline & & & Fusidic acid & $0^{2}$ & $0^{2}$ & n. a. & \\
\hline & & & Gentamicin & $12^{2}$ & $12^{2}$ & n. a. & \\
\hline & & & Imipenem & $15^{2}$ & $11^{2}$ & $\mathrm{R}$ & \\
\hline & & & Rifampicin & $5^{2}$ & $5^{2}$ & n. a. & \\
\hline & & & Tetracycline & $17^{2}$ & $14^{2}$ & $\mathrm{R}$ & \\
\hline & & & Trimethoprim & $13^{2}$ & $0^{2}$ & $\mathrm{R}$ & \\
\hline & & & Vancomycin & $0^{2}$ & $0^{2}$ & n. a. & \\
\hline \multirow{12}{*}{ Escherichia coli } & \multirow{12}{*}{ ATCC 27325} & \multirow{12}{*}{ 4096-fold } & Amoxicillin & $8^{1}$ & $8^{1}$ & n. a. & \multirow{12}{*}{ [52] } \\
\hline & & & Amoxicillin-clavulanic acid & $8^{1}$ & $8^{1}$ & n. a. & \\
\hline & & & Chloramphenicol & $16^{1}$ & $256^{1}$ & $\mathrm{R}$ & \\
\hline & & & Ciprofloxacin & $4^{1}$ & $4^{1}$ & n. a. & \\
\hline & & & Clindamycin & $>256^{1}$ & $>256^{1}$ & n. a. & \\
\hline & & & Colistin sulfate & $16^{1}$ & $16^{1}$ & n. a. & \\
\hline & & & Fusidic acid & $>256^{1}$ & $>256^{1}$ & n. a. & \\
\hline & & & Gentamicin & $8^{1}$ & $8^{1}$ & n. a. & \\
\hline & & & Rifampicin & $256^{1}$ & $256^{1}$ & n. a. & \\
\hline & & & Tetracycline & $32^{1}$ & $32^{1}$ & n. a. & \\
\hline & & & Trimethoprim & $32^{1}$ & $32^{1}$ & n. a. & \\
\hline & & & Vancomycin & $>256^{1}$ & $>256^{1}$ & n. a. & \\
\hline
\end{tabular}


Table 4. Cont.

\begin{tabular}{|c|c|c|c|c|c|c|c|}
\hline Species & Strain(s) & MIC Increase (TRI) & Antibiotic(s) & Pre-Value & Post-Value & Category & Reference \\
\hline Escherichia coli & Strain O55:H7 & 2048-fold & $\begin{array}{c}\text { Amoxicillin } \\
\text { Amoxicillin-clavulanic acid } \\
\text { Chloramphenicol } \\
\text { Ciprofloxacin } \\
\text { Clindamycin } \\
\text { Colistin sulfate } \\
\text { Fusidic acid } \\
\text { Gentamicin } \\
\text { Rifampicin } \\
\text { Tetracycline } \\
\text { Trimethoprim } \\
\text { Vancomycin }\end{array}$ & $\begin{array}{c}8^{1} \\
16^{1} \\
16^{1} \\
2^{1} \\
>256^{1} \\
16^{1} \\
>256^{1} \\
8^{1} \\
>256^{1} \\
32^{1} \\
32^{1} \\
0^{1}\end{array}$ & $\begin{array}{c}8^{1} \\
8^{1} \\
8^{1} \\
2^{1} \\
>256^{1} \\
16^{1} \\
>256^{1} \\
16^{1} \\
>256^{1} \\
32^{1} \\
256^{1} \\
0^{1}\end{array}$ & $\begin{array}{l}\text { n.a. } \\
\text { n.a. } \\
\text { n.a. } \\
\text { n.a. } \\
\text { n.a. } \\
\text { n.a. } \\
\text { n.a. } \\
\text { n.a. } \\
\text { n.a. } \\
\text { n.a. } \\
\text { R } \\
\text { n.a. }\end{array}$ & [52] \\
\hline Escherichia coli & NCTC 12900 & 8192-fold & $\begin{array}{c}\text { Amoxicillin } \\
\text { Amoxicillin-clavulanic acid } \\
\text { Chloramphenicol } \\
\text { Ciprofloxacin } \\
\text { Clindamycin } \\
\text { Colistin sulfate } \\
\text { Fusidic acid } \\
\text { Gentamicin } \\
\text { Rifampicin } \\
\text { Tetracycline } \\
\text { Trimethoprim } \\
\text { Vancomycin }\end{array}$ & $\begin{array}{c}32^{1} \\
4^{1} \\
32^{1} \\
2^{1} \\
>256^{1} \\
8^{1} \\
>256^{1} \\
16^{1} \\
>256^{1} \\
32^{1} \\
64^{1} \\
0^{1}\end{array}$ & $\begin{array}{c}>256^{1} \\
256^{1} \\
256^{1} \\
2^{1} \\
>256^{1} \\
16^{1} \\
>256^{1} \\
16^{1} \\
>256^{1} \\
>256^{1} \\
>256^{1} \\
0^{1}\end{array}$ & $\begin{array}{l}\mathrm{R} \\
\mathrm{R} \\
\mathrm{R} \\
\text { n. } \mathrm{a} . \\
\text { n. } \mathrm{a} . \\
\text { n. } a . \\
\text { n. } a . \\
\text { n. } a . \\
\text { n. } a . \\
\mathrm{R} \\
\mathrm{R} \\
\text { n. } a\end{array}$ & [52] \\
\hline $\begin{array}{c}\text { Fusobacterium } \\
\text { nucleatum }\end{array}$ & ATCC 10953 & None & $\begin{array}{l}\text { Metronidazole } \\
\text { Tetracycline }\end{array}$ & $\begin{array}{l}250^{1} \\
3.9^{1}\end{array}$ & $\begin{array}{l}500^{1} \\
2.9^{1}\end{array}$ & - & [50] \\
\hline Neisseria subflava & Strain A1078 & None & $\begin{array}{l}\text { Metronidazole } \\
\text { Tetracycline }\end{array}$ & $\begin{array}{l}62.5^{1} \\
3.9^{1}\end{array}$ & $\begin{array}{l}52.1^{1} \\
6.8^{1}\end{array}$ & $\begin{array}{l}- \\
-\end{array}$ & {$[50]$} \\
\hline Pantoea agglomerans & $\begin{array}{l}\text { Biocide-sensitive } \\
\text { strain from } \\
\text { organic foods }\end{array}$ & 150-fold & $\begin{array}{c}\text { Ampicillin } \\
\text { Ceftazidime } \\
\text { Sulfamethoxazol }\end{array}$ & $\begin{array}{l}- \\
- \\
-\end{array}$ & $\begin{array}{c}64^{1} \\
64^{1} \\
1024^{1}\end{array}$ & $\begin{array}{l}\mathrm{R} \\
\mathrm{R} \\
\mathrm{R}\end{array}$ & {$[51]$} \\
\hline Pantoea ananatis & $\begin{array}{l}2 \text { biocide-sensitive } \\
\text { strains from } \\
\text { organic foods }\end{array}$ & $\begin{array}{l}\text { 5-fold- } \\
200 \text {-fold }\end{array}$ & $\begin{array}{c}\text { Sulfamethoxazol } \\
\text { Trimethoprim-sulfamethoxazol } \\
\text { Ampicillin } \\
\text { Cefotaxime }\end{array}$ & $\begin{array}{l}- \\
- \\
-\end{array}$ & $\begin{array}{l}1024(2)^{1} \\
8 / 152(2)^{1} \\
32(1)^{1} \\
64(1)^{1}\end{array}$ & $\begin{array}{l}\mathrm{R} \\
\mathrm{R} \\
\mathrm{R} \\
\mathrm{R}\end{array}$ & {$[51]$} \\
\hline
\end{tabular}


Table 4. Cont

\begin{tabular}{|c|c|c|c|c|c|c|c|}
\hline Species & Strain(s) & MIC Increase (TRI) & Antibiotic(s) & Pre-Value & Post-Value & Category & Reference \\
\hline \multirow{3}{*}{ Pantoea spp. } & 2 biocide-sensitive & & Sulfamethoxazol & - & $1024(1)^{1}$ & $\mathrm{R}$ & \multirow{3}{*}{ [51] } \\
\hline & strains from & 2-fold-3-fold & Ceftazidime & - & $64(1)^{1}$ & $\mathrm{R}$ & \\
\hline & organic foods & & Cefotaxime & - & $128(1)^{1}$ & $\mathrm{R}$ & \\
\hline \multirow{2}{*}{$\begin{array}{l}\text { Porphyromonas } \\
\text { gingivalis }\end{array}$} & \multirow{2}{*}{ Strain W50 } & \multirow{2}{*}{ None } & Metronidazole & $31.3^{1}$ & $62.5^{1}$ & - & \multirow{2}{*}{ [50] } \\
\hline & & & Tetracycline & $3.0^{1}$ & $1.0^{1}$ & - & \\
\hline \multirow{2}{*}{ Prevotella nigrescens } & \multirow{2}{*}{ Strain T588 } & \multirow{2}{*}{ 2-fold } & Metronidazole & $62.5^{1}$ & $62.5^{1}$ & - & \multirow{2}{*}{ [50] } \\
\hline & & & Tetracycline & $1.0^{1}$ & $1.0^{1}$ & - & \\
\hline \multirow{6}{*}{ Salmonella spp. } & \multirow{6}{*}{$\begin{array}{l}3 \text { biocide-sensitive } \\
\text { strains from } \\
\text { organic foods }\end{array}$} & \multirow{6}{*}{$\begin{array}{l}\text { 2-fold- } \\
200 \text {-fold }\end{array}$} & Trimethoprim-sulfamethoxazol & - & $8 / 152(2)^{1}$ & $\mathrm{R}$ & \multirow{6}{*}{ [51] } \\
\hline & & & Cefotaxime & - & $64 / 128(2)^{1}$ & $\mathrm{R}$ & \\
\hline & & & Nalidixic acid & - & $64(2)^{1}$ & $\mathrm{R}$ & \\
\hline & & & Ampicillin & - & $64(1)^{1}$ & $\mathrm{R}$ & \\
\hline & & & Sulfamethoxazol & - & $1024(1)^{1}$ & $\mathrm{R}$ & \\
\hline & & & Imipenem & - & $32(1)^{1}$ & $\mathrm{R}$ & \\
\hline \multirow{9}{*}{ Salmonella spp. } & \multirow{9}{*}{$\begin{array}{l}6 \text { strains with } \\
\text { higher MICs to } \\
\text { biocidal products }\end{array}$} & \multirow{9}{*}{$\begin{array}{c}500 \text {-fold- } \\
10.000 \text {-fold (3) }\end{array}$} & Piperacillin & $<4^{3}$ & $16^{3}$ & I & \multirow{9}{*}{ [16] } \\
\hline & & & Ceftiofur & $2^{3}$ & $>8^{3}$ & $\mathrm{R}$ & \\
\hline & & & Amikacin & $4^{3}$ & $16^{3}$ & I & \\
\hline & & & Gentamicin & $<1^{3}$ & $4^{3}$ & I & \\
\hline & & & Kanamycin & $<8^{3}$ & $32^{3}$ & I & \\
\hline & & & Chloramphenicol & $4^{3}$ & $16^{3}$ & I & \\
\hline & & & Cefoxitin & $16^{3}$ & $32^{3}$ & $\mathrm{R}$ & \\
\hline & & & Nalidixic acid & $8^{3}$ & $32^{3}$ & $\mathrm{R}$ & \\
\hline & & & Sulfisoxazole & $32^{3}$ & $>256^{3}$ & I & \\
\hline \multirow{2}{*}{ Veillonella dispar } & \multirow{2}{*}{ ATCC 17745} & \multirow{2}{*}{ None } & Metronidazole & $78.1^{1}$ & $31.3^{1}$ & - & \multirow{2}{*}{ [50] } \\
\hline & & & Tetracycline & $31.3^{1}$ & $27.4^{1}$ & - & \\
\hline
\end{tabular}

${ }^{1}$ microdilution method $(\mathrm{mg} / \mathrm{L}) ;{ }^{2}$ disc diffusion test $(\mathrm{mm}) ;{ }^{3}$ NARMS plates $(\mathrm{mg} / \mathrm{L}) ;{ }^{-}{ }^{\prime \prime}$ = no information; $\mathrm{R}=$ resistant; $\mathrm{I}=$ intermediate susceptible; $\mathrm{S}=$ susceptible; $\mathrm{n}$. a. = not applicable; ()$=$ number of strains, isolates or experiments; (P1) = passage $1 ;(\mathrm{P} 2)=$ passage 2 . 


\subsection{Increase of Horizontal Gene Transfer}

Horizontal transfer of mobile antibiotic resistance elements by conjugation could be significantly increased by low level exposure to triclosan $(0.1 \mathrm{mg} / \mathrm{L})$ to a recipient Escherichia coli strain [34].

\subsection{Additional Findings}

Triclosan is also a biocidal agent which can enhance resistance to antibiotics in some Gram-negative species. An associated cross-tolerance or cross-resistance between triclosan and various antibiotics seems uncommon in Acinetobacter johnsonii and Escherichia coli [53] although one study has described a cross-tolerance between triclosan and chloramphenicol (intermediate susceptibility) in an Acinetobacter johnsonii strain [54]. Among 52 Pseudomonas spp. isolates from meat chain production, a general cross-tolerance between triclosan and ampicillin, amoxicillin, erythromycin, imipenem and trimethoprim was described [22]. Resistance in Salmonella caused by increasing concentrations of triclosan is associated with an overexpression of the AcrAB efflux pump [23]. A possible mechanism was shown with Agrobacterium tumefaciens where triclosan abolishes the interaction between the transcriptional repressor of the acrABR operon (acrR) and the DNA to which acrR specifically binds in the acrA promoter region [55]. A correlation between a decreased triclosan susceptibility and multidrug-resistance was shown in 428 Salmonella enterica isolates. Four percent of the isolates were triclosan-tolerant, $56 \%$ of them were multidrug-resistant. Among the remaining triclosan-sensitive isolates only $12 \%$ were multidrug-resistant [56]. Efflux pumps were also considered to explain a lower susceptibility to triclosan in antibiotic-resistant Escherichia coli and Salmonella spp. isolated from poultry and clinical samples [57]. In the domestic setting no antibiotic and antibacterial agent cross-resistance in target bacteria from antibacterial product users and nonusers was found [58].

\section{Didecyldimethylammonium Chloride}

\subsection{Antibiotic Tolerance or Resistance after Low Level Biocide Exposure}

One study was found with data for 2 species (Table 5). 59\% of 54 Escherichia coli strains became multiresistant to antibiotics after low level didecyldimethylammonium chloride exposure whereas a new resistance to at least one antibiotic occurred in only 13\% of 54 Salmonella enterica strains.

Table 5. Gram-negative species with antibiotic tolerance or resistance after low level exposure (<MIC value) to didecyldimethylammonium chloride (DDAC).

\begin{tabular}{cccc}
\hline Species & Strain(s) & $\begin{array}{c}\text { Type of DDAC } \\
\text { Exposure }\end{array}$ & Antibiotic(s) \\
\hline Escherichia coli & $\begin{array}{c}54 \text { strains from pig } \\
\text { faeces or pork meat }\end{array}$ & $\begin{array}{c}7 \text { d at various } \\
\text { concentrations. }\end{array}$ & $\begin{array}{c}32 \text { strains became multiresistant, most } \\
\text { of them with a new resistance }{ }^{1} \text { to } \\
\text { chloramphenicol, ampicillin, } \\
\text { cefotaxime, ceftazidime } \\
\text { and ciprofloxacin }\end{array}$ \\
\hline Salmonella enterica & $\begin{array}{c}54 \text { strains from pig } \\
\text { faeces or pork meat }\end{array}$ & $\begin{array}{c}7 \text { d at various } \\
\text { concentrations }\end{array}$ & $\begin{array}{c}\text { 7 strains acquired a new resistance }{ }^{1}, \\
\text { mainly to chloramphenicol (3 strains) }\end{array}$ \\
\hline & & 1 microdilution method (mg/L).
\end{tabular}

\subsection{Additional Findings}

Fewer data are available with didecyldimethylammonium chloride. Some studies describe a cross-tolerance between didecyldimethylammonium chloride and antibiotics. For example, in $153 \mathrm{E}$. coli blood culture isolates a higher MIC of didecyldimethylammonium chloride was associated with a decreased susceptibility to cotrimoxazole [21]. In E.coli didecyldimethylammonium chloride-MICs were positively correlated with MICs of piperacillin and sulphamethoxazole-trimethoprim [60]. However exposure of A. baumannii, C. sakazakii, E. coli, 
P. aeruginosa and P. putida to increasing didecyldimethylammonium chloride concentrations over 14 passages of four days each did not result in antibiotic resistance [61].

\section{Sodium Hypochlorite}

\subsection{Antibiotic Tolerance or Resistance after Low Level Biocide Exposure}

Some strains of Salmonella species, adapted to sodium hypochlorite, have occasionally developed an associated resistance to specific antibiotics such as gentamicin in S. Anatum, ceftazidime in S. Enteritidis, amikacin, ampicillin, chloramphenicol and nitrafurantoin in S. Hadar, gentamicin, ceftazidime, amikacin, tobramycin, cefoxitin, and tetracycline in S. Infantis, amikacin and ampicillin/sulbactam in S. Kentucky, gentamicin, ceftazidime, tobramycin, cefoxitin, cefazolin and nalidixic acid in S. Thompson, amikacin, tobramycin, cefazolin, cefotaxime in S. Thyphimurium, teicoplanin in S. Virchow, and gentamicin, nitrafurantoin, cephalothin, cefepime and enrofloxacin in Salmonella spp. strain 1,4, [5],12:i- [62]. It is particularly interesting that an E. coli strain was found to be viable but non-culturable after low level exposure to sodium hypochlorite and that the same adapted cells were able to better persist in the presence of various antibiotics [63].

\subsection{Effect on Antibiotic Resistance Genes}

Sodium hypochlorite can reduce antibiotic resistance genes or plasmids to some extent (mostly $\leq 1.0 \mathrm{log}$ ). This effect has been shown with three antibiotic resistance genes (sul1, blaTEM, blaCTX-M) which were reduced by $0.8-0.9 \mathrm{log}$. The antibiotic resistance plasmid pB10 from an E. coli strain was also reduced by $1.0 \log$ [64]. A somatic coliphage could be reduced in $30 \mathrm{~min}$ by at least $1.0 \mathrm{log}$. The antibiotic resistance genes, however, were not significantly reduced $(0.2-0.6 \log )$ [65]. Similar findings were reported with the tet(W) gene in Acinetobacter, Aeromonas, Chryseobacterium, E. coli, Pseudomonas and Serratia. It was mostly reduced by 0.0-0.9 log immediately after exposure to sodium hypochlorite, the effect was stronger in Acinetobacter (1.8 log) and Chryseobacterium (4.0 log) [66]. A higher concentration of active chlorine (range: $2-32 \mathrm{mg} / \mathrm{L}$ ) decreases the abundance of antibiotic resistance genes in wastewater linearly [67]. Bacteria may, however, persist after sodium hypochlorite treatment. Survivors may outgrow from the biofilm which may increase the level of antibiotic resistance genes in water. Sodium hypochlorite at $1 \mathrm{mg} / \mathrm{L}$ can destroy the piperazine ring of ciprofloxacin in drinking water distribution systems. As a consequence, specific antibiotic resistance genes increased in effluents (e.g., mexA and qnrS) and others increased in biofilms (qnrA and qnrB). These bacterial genera seem to grow by transformation of ciprofloxacin chlorination products in drinking water distribution systems [68].

\section{Other Biocidal Agents}

\subsection{Antibiotic Tolerance or Resistance after Low Level Biocide Exposure}

Cross-tolerance between octenidine and gentamicin, colistin, amikacin, and tobramycin has been described in a Pseudomonas aeruginosa isolate [69]. No cross-tolerance or cross-resistance to antibiotics has so far been described after low level exposure to ethanol, propanol, peracetic acid, polyhexanide, povidone iodine, glutaraldehyde, and hydrogen peroxide.

\subsection{Effect on Antibiotic Resistance Genes}

The data for peracetic acid are not so clear [70]. Peracetic acid in waste water was shown to stimulate the selection of antibiotic resistance genes [71]. It was, however, not able to reduce nine antibiotic resistance genes (ampC, mecA, ermB, sul1, sul2, tetA, tetO, tetW, vanA) in wastewater [72]. For triclosan, didecyldimethylammonium chloride, povidone iodine, octenidine, polyhexanide, glutaraldehyde, hydrogen peroxide, ethanol, and propanol no data on a possible induction of antibiotic resistance genes or a reduction of antibiotic resistance genes were found. 


\subsection{Increase of Horizontal Gene Transfer}

Production of hydrogen peroxide in cells of Streptococcus gordonii was shown to cause release of extracellular DNA which may serve as a pool for novel genetic traits such as antimicrobial resistance [73]. Hydrogen peroxide produced by one species is able to induce the DNA release by another which has important implications for the role of hydrogen peroxide in interspecies horizontal gene transfer [73]. Whether this finding has any relevance for extracellular low-level hydrogen peroxide exposure is unknown. Low level chlorination of $0.3-0.5 \mathrm{mg} / \mathrm{L}$ chlorine was able to decrease conjugative transfer of the RP4 plasmid in drinking water [74]. No effect on horizontal gene transfer by low-level exposure was so far described for ethanol, propanol, peracetic acid, glutaraldehyde, polihexanide, DDAC, octenidine, and povidone iodine.

\subsection{Additional Findings}

Hydrogen peroxide and peracetic acid were not among the biocidal agents with evidence that low level exposure can cause antibiotic resistance. This is probably explained by their lower stability which may make it more difficult for bacteria to adapt to the biocidal agents. Another advantage for peracetic acid in this context is that it was able to transform different beta-lactam antibiotics in wastewater at concentrations of $0.0005-0.002 \%$ which may help to reduce antibiotic selection pressure in wastewater [75].

\section{Discussion}

The health burden of five types of infection with antibiotic-resistant bacteria is high in Europe with an estimated 671,689 infections in 2015, of which $63.5 \%$ were associated with healthcare [76]. Antibiotic resistance caused by some biocidal agents is very likely of minor relevance in this context. But nevertheless it seems necessary to critically review disinfectant formulations with the aim to ban any unnecessary selection pressure.

One example is alcohol-based hand rubs. Some products contain in addition to the alcohol(s) non-volatile biocidal agents such as chlorhexidine digluconate, triclosan, benzalkonium chloride, didecyldimethylammonium chloride, polihexanide, or octenidine dihydrochloride [77]. A recent review with some of the agents shows that all formulations containing such an additional biocidal agent fail to show a superior bactericidal efficacy according to EN 12791 after three hours under the surgical glove [78]. In addition, a health benefit (e.g., reduction of surgical site infection) has so far not been shown for any of the additional biocidal agents in alcohol-based hand rubs [79]. Taking into account that there is no health benefit for any of these additional biocidal agents for the application hand disinfection but a realistic potential to enhance the development of antibiotic resistance it seems logical and responsible to prefer alcohol-based hand rubs without additional biocidal agents as long as they have an equivalent user acceptability and efficacy for hand disinfection ("antiseptic stewardship") [77]. The Commission for Hospital Hygiene and Infection Control (KRINKO) at the Robert Koch-Institute, Berlin, Germany, has therefore recommended that alcohol-based hand rubs with persistent biocidal agents cannot be recommended [80].

Additional biocidal agents in alcohol-based skin antiseptics should also be reviewed. Some products contain chlorhexidine, octenidine, povidone iodine, or benzalkonium chloride [81]. A proven health benefit (prevention of catheter-associated bloodstream infections and probably also surgical site infections) has so far only been shown for the additional chlorhexidine [82-86]. Additional octenidine may also have a health benefit for the prevention of catheter-associated bloodstream infections [87]. No health benefit has been shown for additional benzalkonium chloride or povidone iodine. For benzalkonium chloride at a low concentration there is even evidence that a persistent antimicrobial effect on the skin over $48 \mathrm{~h}$ is lacking [88]. The use of chlorhexidine in alcohol-based skin antiseptics seems reasonable despite some risks. The use of octenidine in alcohol-based skin antiseptics may also be favourable although the evidence for a health benefit is sparse. Benzalkonium chloride in 
alcohol-based skin antiseptics does not have any health benefit but has some relevant risks including antibiotic resistance development. It should be replaced [81].

For other types of applications such as surface disinfection, wound antisepsis, mucous membrane antisepsis, or instrument disinfection, preference should be given to those biocidal agents without or with a low selection pressure assuming that their antimicrobial activity, material compatibility, and user safety is at least as good for the intended use. Other antimicrobial agents such as cold plasma may be an alternative in the future [89].

\section{Conclusions}

Antibiotic resistance may occur after exposure of various Gram-negative species to sublethal concentrations of some biocidal agents such as benzalkonium chloride, chlorhexidine or triclosan. Their use as an antiseptic agent should be restricted to applications with a proven health benefit. General preference should be given to biocidal agents without or with a low selection pressure assuming that their antimicrobial activity, material compatibility, and user safety is at least as good for the intended use.

Funding: This research received no external funding.

Conflicts of Interest: The author was employed until 2016 by Bode Chemie GmbH, Hamburg, Germany, a manufacturer of disinfectants.

\section{References}

1. Jones, R. Bacterial resistance and topical antimicrobial wash products. Am. J. Infect. Control 1999, 27, 351-363. [CrossRef]

2. Department of Health and Human Services, Food and Drug Administration. Safety and effectiveness of consumer antiseptics; topical antimicrobial drug products for over-the-counter human use. Fed. Reg. 2016, 81, 61106-61130.

3. McNamara, P.J.; Levy, S.B. Triclosan: An instructive tale. Antimicrob. Agents Chemother. 2016, 60, 7015-7016. [CrossRef] [PubMed]

4. Kampf, G. Introduction. In Antiseptic Stewardship: Biocide Resistance and Clinical Implications; Kampf, G., Ed.; Springer International Publishing: Cham, Switzerland, 2018; pp. 1-7.

5. Soumet, C.; Fourreau, E.; Legrandois, P.; Maris, P. Resistance to phenicol compounds following adaptation to quaternary ammonium compounds in Escherichia coli. Vet. Microbiol. 2012, 158, 147-152. [CrossRef] [PubMed]

6. Knapp, L.; Rushton, L.; Stapleton, H.; Sass, A.; Stewart, S.; Amezquita, A.; McClure, P.; Mahenthiralingam, E.; Maillard, J.Y. The effect of cationic microbicide exposure against Burkholderia cepacia complex (bcc); the use of Burkholderia lata strain 383 as a model bacterium. J. Appl. Microbiol. 2013, 115, 1117-1126. [CrossRef] [PubMed]

7. Gadea, R.; Fernandez Fuentes, M.A.; Perez Pulido, R.; Galvez, A.; Ortega, E. Effects of exposure to quaternary-ammonium-based biocides on antimicrobial susceptibility and tolerance to physical stresses in bacteria from organic foods. Food Microbiol. 2017, 63, 58-71. [CrossRef] [PubMed]

8. Langsrud, S.; Sundheim, G.; Holck, A.L. Cross-resistance to antibiotics of Escherichia coli adapted to benzalkonium chloride or exposed to stress-inducers. J. Appl. Microbiol. 2004, 96, 201-208. [CrossRef] [PubMed]

9. Bore, E.; Hebraud, M.; Chafsey, I.; Chambon, C.; Skjaeret, C.; Moen, B.; Moretro, T.; Langsrud, O.; Rudi, K.; Langsrud, S. Adapted tolerance to benzalkonium chloride in Escherichia coli K-12 studied by transcriptome and proteome analyses. Microbiology 2007, 153, 935-946. [CrossRef]

10. Braoudaki, M.; Hilton, A.C. Adaptive resistance to biocides in Salmonella enterica and Escherichia coli O157 and cross-resistance to antimicrobial agents. J. Clin. Microbiol. 2004, 42, 73-78. [CrossRef]

11. Nhung, N.T.; Thuy, C.T.; Trung, N.V.; Campbell, J.; Baker, S.; Thwaites, G.; Hoa, N.T.; Carrique-Mas, J. Induction of antimicrobial resistance in Escherichia coli and non-typhoidal Salmonella strains after adaptation to disinfectant commonly used on farms in Vietnam. Antibiotics 2015, 4, 480-494. [CrossRef] 
12. Pagedar, A.; Singh, J.; Batish, V.K. Efflux mediated adaptive and cross resistance to ciprofloxacin and benzalkonium chloride in Pseudomonas aeruginosa of dairy origin. J. Basic Microbiol. 2011, 51, 289-295. [CrossRef] [PubMed]

13. Joynson, J.A.; Forbes, B.; Lambert, R.J. Adaptive resistance to benzalkonium chloride, amikacin and tobramycin: The effect on susceptibility to other antimicrobials. J. Appl. Microbiol. 2002, 93, 96-107. [CrossRef] [PubMed]

14. Mc Cay, P.H.; Ocampo-Sosa, A.A.; Fleming, G.T. Effect of subinhibitory concentrations of benzalkonium chloride on the competitiveness of Pseudomonas aeruginosa grown in continuous culture. Microbiology 2010, 156, 30-38. [CrossRef] [PubMed]

15. Kim, M.; Weigand, M.R.; Oh, S.; Hatt, J.K.; Krishnan, R.; Tezel, U.; Pavlostathis, S.G.; Konstantinidis, K.T. Widely used benzalkonium chloride disinfectants can promote antibiotic resistance. Appl. Environ. Microbiol. 2018, 84, e01201-18. [CrossRef] [PubMed]

16. Condell, O.; Iversen, C.; Cooney, S.; Power, K.A.; Walsh, C.; Burgess, C.; Fanning, S. Efficacy of biocides used in the modern food industry to control Salmonella enterica, and links between biocide tolerance and resistance to clinically relevant antimicrobial compounds. Appl. Environ. Microbiol. 2012, 78, 3087-3097. [CrossRef] [PubMed]

17. Slifierz, M.J.; Friendship, R.M.; Weese, J.S. Methicillin-resistant Staphylococcus aureus in commercial swine herds is associated with disinfectant and zinc usage. Appl. Environ. Microbiol. 2015, 81, 2690-2695. [CrossRef] [PubMed]

18. Heir, E.; Langsrud, S.; Sidhu, M.S.; Steinbakk, M. [can disinfectants contribute to antibiotic resistance?]. Tidsskr. Nor. Laegeforen. 2001, 121, 3201-3206. [PubMed]

19. Jiang, X.; Xu, Y.; Li, Y.; Zhang, K.; Liu, L.; Wang, H.; Tian, J.; Ying, H.; Shi, L.; Yu, T. Characterization and horizontal transfer of qacH-associated class 1 integrons in Escherichia coli isolated from retail meats. Int. J. Food Microbiol. 2017, 258, 12-17. [CrossRef]

20. Morita, Y.; Murata, T.; Mima, T.; Shiota, S.; Kuroda, T.; Mizushima, T.; Gotoh, N.; Nishino, T.; Tsuchiya, T. Induction of mexCD-oprJ operon for a multidrug efflux pump by disinfectants in wild-type Pseudomonas aeruginosa PAO1. J. Antimicrob. Chemother. 2003, 51, 991-994. [CrossRef]

21. Buffet-Bataillon, S.; Branger, B.; Cormier, M.; Bonnaure-Mallet, M.; Jolivet-Gougeon, A. Effect of higher minimum inhibitory concentrations of quaternary ammonium compounds in clinical E. coli isolates on antibiotic susceptibilities and clinical outcomes. J. Hosp. Infect. 2011, 79, 141-146. [CrossRef]

22. Lavilla Lerma, L.; Benomar, N.; Casado Munoz Mdel, C.; Galvez, A.; Abriouel, H. Correlation between antibiotic and biocide resistance in mesophilic and psychrotrophic Pseudomonas spp. isolated from slaughterhouse surfaces throughout meat chain production. Food Microbiol. 2015, 51, 33-44. [CrossRef] [PubMed]

23. Karatzas, K.A.; Webber, M.A.; Jorgensen, F.; Woodward, M.J.; Piddock, L.J.; Humphrey, T.J. Prolonged treatment of Salmonella enterica serovar typhimurium with commercial disinfectants selects for multiple antibiotic resistance, increased efflux and reduced invasiveness. J. Antimicrob. Chemother. 2007, 60, 947-955. [CrossRef] [PubMed]

24. Chuanchuen, R.; Khemtong, S.; Padungtod, P. Occurrence of qacE/qacEDelta1 genes and their correlation with class 1 integrons in Salmonella enterica isolates from poultry and swine. Southeast Asian J. Trop. Med. Public Health 2007, 38, 855-862. [PubMed]

25. Kucken, D.; Feucht, H.; Kaulfers, P. Association of qacE and qacEDelta1 with multiple resistance to antibiotics and antiseptics in clinical isolates of gram-negative bacteria. FEMS Microbiol. Lett. 2000, 183, 95-98. [CrossRef]

26. Law, C.J.; Alegre, K.O. Clamping down on drugs: The Escherichia coli multidrug efflux protein MdtM. Res. Microbiol. 2018, 169, 461-467. [CrossRef] [PubMed]

27. Bay, D.C.; Stremick, C.A.; Slipski, C.J.; Turner, R.J. Secondary multidrug efflux pump mutants alter Escherichia coli biofilm growth in the presence of cationic antimicrobial compounds. Res. Microbiol. 2017, 168, 208-221. [CrossRef] [PubMed]

28. Nagai, K.; Murata, T.; Ohta, S.; Zenda, H.; Ohnishi, M.; Hayashi, T. Two different mechanisms are involved in the extremely high-level benzalkonium chloride resistance of a Pseudomonas fluorescens strain. Microbiol. Immunol. 2003, 47, 709-715. [CrossRef]

29. Guo, W.; Cui, S.; Xu, X.; Wang, H. Resistant mechanism study of benzalkonium chloride selected Salmonella typhimurium mutants. Microb. Drug Resist. 2014, 20, 11-16. [CrossRef] 
30. Pumbwe, L.; Skilbeck, C.A.; Wexler, H.M. Induction of multiple antibiotic resistance in Bacteroides fragilis by benzene and benzene-derived active compounds of commonly used analgesics, antiseptics and cleaning agents. J. Antimicrob. Chemother. 2007, 60, 1288-1297. [CrossRef]

31. Gadea, R.; Glibota, N.; Perez Pulido, R.; Galvez, A.; Ortega, E. Adaptation to biocides cetrimide and chlorhexidine in bacteria from organic foods: Association with tolerance to other antimicrobials and physical stresses. J. Agric. Food Chem. 2017, 65, 1758-1770. [CrossRef]

32. Wesgate, R.; Grasha, P.; Maillard, J.Y. Use of a predictive protocol to measure the antimicrobial resistance risks associated with biocidal product usage. Am. J. Infect. Control 2016, 44, 458-464. [CrossRef]

33. Wand, M.E.; Bock, L.J.; Bonney, L.C.; Sutton, J.M. Mechanisms of increased resistance to chlorhexidine and cross-resistance to colistin following exposure of Klebsiella pneumoniae clinical isolates to chlorhexidine. Antimicrob. Agents Chemother. 2017, 61, e01162-16. [CrossRef] [PubMed]

34. Jutkina, J.; Marathe, N.P.; Flach, C.F.; Larsson, D.G.J. Antibiotics and common antibacterial biocides stimulate horizontal transfer of resistance at low concentrations. Sci. Total Environ. 2017, 616-617, 172-178. [CrossRef] [PubMed]

35. Ciusa, M.L.; Furi, L.; Knight, D.; Decorosi, F.; Fondi, M.; Raggi, C.; Coelho, J.R.; Aragones, L.; Moce, L.; Visa, P.; et al. A novel resistance mechanism to triclosan that suggests horizontal gene transfer and demonstrates a potential selective pressure for reduced biocide susceptibility in clinical strains of Staphylococcus aureus. Int. J. Antimicrob. Agents 2012, 40, 210-220. [CrossRef] [PubMed]

36. Fraud, S.; Campigotto, A.J.; Chen, Z.; Poole, K. MexCD-OprJ multidrug efflux system of Pseudomonas aeruginosa: Involvement in chlorhexidine resistance and induction by membrane-damaging agents dependent upon the AlgU stress response sigma factor. Antimicrob. Agents Chemother. 2008, 52, 4478-4482. [CrossRef] [PubMed]

37. Maris, P. [resistance of 700 gram-negative bacterial strains to antiseptics and antibiotics]. Ann. Res. Vet. 1991, $22,11-23$.

38. Fernandez-Cuenca, F.; Tomas, M.; Caballero-Moyano, F.J.; Bou, G.; Martinez-Martinez, L.; Vila, J.; Pachon, J.; Cisneros, J.M.; Rodriguez-Bano, J.; Pascual, A. Reduced susceptibility to biocides in Acinetobacter baumannii: Association with resistance to antimicrobials, epidemiological behaviour, biological cost and effect on the expression of genes encoding porins and efflux pumps. J. Antimicrob. Chemother. 2015, 70, 3222-3229. [CrossRef]

39. Gajadhar, T.; Lara, A.; Sealy, P.; Adesiyun, A.A. Microbial contamination of disinfectants and antiseptics in four major hospitals in trinidad. Rev. Panam. Salud Publica 2003, 14, 193-200. [CrossRef]

40. Tattawasart, U.; Maillard, J.-Y.; Furr, J.R.; Russell, A.D. Outer membrane changes in Pseudomonas stutzeri resistant to chlorhexidine diacetate and cetylpyridinium chloride. Int. J. Antimicrob. Agents 2000, 16, $233-238$. [CrossRef]

41. Tattawasart, U.; Maillard, J.-Y.; Furr, J.R.; Russell, A.D. Development of resistance to chlorhexidine diacetate and cetylpyridinium chloride in Pseudomonas stutzeri and changes in antibiotic susceptibility. J. Hosp. Infect. 1999, 42, 219-229. [CrossRef]

42. Rose, H.; Baldwin, A.; Dowson, C.G.; Mahenthiralingam, E. Biocide susceptibility of the Burkholderia cepacia complex. J. Antimicrob. Chemother. 2009, 63, 502-510. [CrossRef] [PubMed]

43. Beier, R.C.; Anderson, P.N.; Hume, M.E.; Poole, T.L.; Duke, S.E.; Crippen, T.L.; Sheffield, C.L.; Caldwell, D.J.; Byrd, J.A.; Anderson, R.C.; et al. Characterization of Salmonella enterica isolates from turkeys in commercial processing plants for resistance to antibiotics, disinfectants, and a growth promoter. Foodborne Pathog. Dis. 2011, 8, 593-600. [CrossRef] [PubMed]

44. Russell, A.D. Do biocides select for antibiotic resistance? J. Pharm. Pharmacol. 2000, 52, 227-233. [CrossRef] [PubMed]

45. Russell, A.D.; Tattawasart, U.; Maillard, J.Y.; Furr, J.R. Possible link between bacterial resistance and use of antibiotics and biocides. Antimicrob. Agents Chemother. 1998, 42, 2151. [CrossRef]

46. Oggioni, M.R.; Coelho, J.R.; Furi, L.; Knight, D.R.; Viti, C.; Orefici, G.; Martinez, J.L.; Freitas, A.T.; Coque, T.M.; Morrissey, I. Significant differences characterise the correlation coefficients between biocide and antibiotic susceptibility profiles in Staphylococcus aureus. Curr. Pharm. Des. 2015, 21, 2054-2057. [CrossRef]

47. Lambert, R.J. Comparative analysis of antibiotic and antimicrobial biocide susceptibility data in clinical isolates of methicillin-sensitive Staphylococcus aureus, methicillin-resistant Staphylococcus aureus and Pseudomonas aeruginosa between 1989 and 2000. J. Appl. Microbiol. 2004, 97, 699-711. [CrossRef] 
48. Russell, A.D. Introduction of biocides into clinical practice and the impact on antibiotic-resistant bacteria. In Symposium Series (Society for Applied Microbiology); National Center for Biotechnology Information, U.S. National Library of Medicine: Bethesda, MD, USA, 2002.

49. Sonbol, F.I.; El-Banna, T.E.; Abd El-Aziz, A.A.; El-Ekhnawy, E. Impact of triclosan adaptation on membrane properties, efflux and antimicrobial resistance of Escherichia coli clinical isolates. J. Appl. Microbiol. 2018. [CrossRef]

50. McBain, A.J.; Ledder, R.G.; Sreenivasan, P.; Gilbert, P. Selection for high-level resistance by chronic triclosan exposure is not universal. J. Antimicrob. Chemother. 2004, 53, 772-777. [CrossRef]

51. Gadea, R.; Fernández Fuentes, M.A.; Pérez Pulido, R.; Gálvez, A.; Ortega, E. Adaptive tolerance to phenolic biocides in bacteria from organic foods: Effects on antimicrobial susceptibility and tolerance to physical stresses. Food Res. Int. 2016, 85, 131-143. [CrossRef]

52. Braoudaki, M.; Hilton, A.C. Low level of cross-resistance between triclosan and antibiotics in Escherichia coli K-12 and E. coli O55 compared to E. coli O157. FEMS Microbiol. Lett. 2004, 235, 305-309. [CrossRef]

53. Cottell, A.; Denyer, S.P.; Hanlon, G.W.; Ochs, D.; Maillard, J.Y. Triclosan-tolerant bacteria: Changes in susceptibility to antibiotics. J. Hosp. Infect. 2009, 72, 71-76. [CrossRef] [PubMed]

54. Lear, J.C.; Maillard, J.Y.; Dettmar, P.W.; Goddard, P.A.; Russell, A.D. Chloroxylenol- and triclosan-tolerant bacteria from industrial sources-Susceptibility to antibiotics and other biocides. Int. Biodeterior. Biodegrad. 2006, 57, 51-56. [CrossRef]

55. Nuonming, P.; Khemthong, S.; Dokpikul, T.; Sukchawalit, R.; Mongkolsuk, S. Characterization and regulation of AcrABR, a RND-type multidrug efflux system, in Agrobacterium tumefaciens C58. Microbiol. Res. 2018, 214, 146-155. [CrossRef] [PubMed]

56. Copitch, J.L.; Whitehead, R.N.; Webber, M.A. Prevalence of decreased susceptibility to triclosan in Salmonella enterica isolates from animals and humans and association with multiple drug resistance. Int. J. Antimicrob. Agents 2010, 36, 247-251. [CrossRef] [PubMed]

57. Thorrold, C.A.; Letsoalo, M.E.; Duse, A.G.; Marais, E. Efflux pump activity in fluoroquinolone and tetracycline resistant Salmonella and E. coli implicated in reduced susceptibility to household antimicrobial cleaning agents. Int. J. Food Microbiol. 2007, 113, 315-320. [CrossRef]

58. Cole, E.C.; Addison, R.M.; Rubino, J.R.; Leese, K.E.; Dulaney, P.D.; Newell, M.S.; Wilkins, J.; Gaber, D.J.; Wineinger, T.; Criger, D.A. Investigation of antibiotic and antibacterial agent cross-resistance in target bacteria from homes of antibacterial product users and nonusers. J. Appl. Microbiol. 2003, 95, 664-676. [CrossRef] [PubMed]

59. Soumet, C.; Meheust, D.; Pissavin, C.; Le Grandois, P.; Fremaux, B.; Feurer, C.; Le Roux, A.; Denis, M.; Maris, P. Reduced susceptibilities to biocides and resistance to antibiotics in food-associated bacteria following exposure to quaternary ammonium compounds. J. Appl. Microbiol. 2016, 121, 1275-1281. [CrossRef]

60. Wieland, N.; Boss, J.; Lettmann, S.; Fritz, B.; Schwaiger, K.; Bauer, J.; Holzel, C.S. Susceptibility to disinfectants in antimicrobial-resistant and -susceptible isolates of Escherichia coli, Enterococcus faecalis and Enterococcus faecium from poultry-ESBL/AmpC-phenotype of E. coli is not associated with resistance to a quaternary ammonium compound, DDAC. J. Appl. Microbiol. 2017, 122, 1508-1517.

61. Forbes, S.; Knight, C.G.; Cowley, N.L.; Amezquita, A.; McClure, P.; Humphreys, G.; McBain, A.J. Variable effects of exposure to formulated microbicides on antibiotic susceptibility in firmicutes and proteobacteria. Appl. Environ. Microbiol. 2016, 82, 3591-3598. [CrossRef]

62. Molina-González, D.; Alonso-Calleja, C.; Alonso-Hernando, A.; Capita, R. Effect of sub-lethal concentrations of biocides on the susceptibility to antibiotics of multi-drug resistant Salmonella enterica strains. Food Control 2014, 40, 329-334. [CrossRef]

63. Lin, H.; Ye, C.; Chen, S.; Zhang, S.; Yu, X. Viable but non-culturable E. coli induced by low level chlorination have higher persistence to antibiotics than their culturable counterparts. Environ. Pollut. 2017, 230, 242-249. [CrossRef] [PubMed]

64. Oh, J.; Salcedo, D.E.; Medriano, C.A.; Kim, S. Comparison of different disinfection processes in the effective removal of antibiotic-resistant bacteria and genes. J. Environ. Sci. 2014, 26, 1238-1242. [CrossRef]

65. Calero-Caceres, W.; Muniesa, M. Persistence of naturally occurring antibiotic resistance genes in the bacteria and bacteriophage fractions of wastewater. Water Res. 2016, 95, 11-18. [CrossRef] [PubMed] 
66. Sullivan, B.A.; Vance, C.C.; Gentry, T.J.; Karthikeyan, R. Effects of chlorination and ultraviolet light on environmental tetracycline-resistant bacteria and tet(W) in water. J. Environ. Chem. Eng. 2017, 5, 777-784. [CrossRef]

67. Zheng, J.; Su, C.; Zhou, J.; Xu, L.; Qian, Y.; Chen, H. Effects and mechanisms of ultraviolet, chlorination, and ozone disinfection on antibiotic resistance genes in secondary effluents of municipal wastewater treatment plants. Chem. Eng. J. 2017, 317, 309-316. [CrossRef]

68. Wang, H.; Hu, C.; Liu, L.; Xing, X. Interaction of ciprofloxacin chlorination products with bacteria in drinking water distribution systems. J. Hazard. Mater. 2017, 339, 174-181. [CrossRef] [PubMed]

69. Shepherd, M.J.; Moore, G.; Wand, M.E.; Sutton, J.M.; Bock, L.J. Pseudomonas aeruginosa adapts to octenidine in the laboratory and a simulated clinical setting, leading to increased tolerance to chlorhexidine and other biocides. J. Hosp. Infect. 2018, 100, e23-e29. [CrossRef]

70. Biswal, B.K.; Khairallah, R.; Bibi, K.; Mazza, A.; Gehr, R.; Masson, L.; Frigon, D. Impact of UV and peracetic acid disinfection on the prevalence of virulence and antimicrobial resistance genes in uropathogenic Escherichia coli in wastewater effluents. Appl. Environ. Microbiol. 2014, 80, 3656-3666. [CrossRef] [PubMed]

71. Di Cesare, A.; Fontaneto, D.; Doppelbauer, J.; Corno, G. Fitness and recovery of bacterial communities and antibiotic resistance genes in urban wastewaters exposed to classical disinfection treatments. Environ. Sci. Technol. 2016, 50, 10153-10161. [CrossRef] [PubMed]

72. Luprano, M.L.; De Sanctis, M.; Del Moro, G.; Di Iaconi, C.; Lopez, A.; Levantesi, C. Antibiotic resistance genes fate and removal by a technological treatment solution for water reuse in agriculture. Sci. Total Environ. 2016, 571, 809-818. [CrossRef] [PubMed]

73. Itzek, A.; Zheng, L.; Chen, Z.; Merritt, J.; Kreth, J. Hydrogen peroxide-dependent DNA release and transfer of antibiotic resistance genes in Streptococcus gordonii. J. Bacteriol. 2011, 193, 6912-6922. [CrossRef] [PubMed]

74. Lin, W.; Li, S.; Zhang, S.; Yu, X. Reduction in horizontal transfer of conjugative plasmid by UV irradiation and low-level chlorination. Water Res. 2016, 91, 331-338. [CrossRef] [PubMed]

75. Zhang, K.; Zhou, X.; Du, P.; Zhang, T.; Cai, M.; Sun, P.; Huang, C.H. Oxidation of beta-lactam antibiotics by peracetic acid: Reaction kinetics, product and pathway evaluation. Water Res. 2017, 123, 153-161. [CrossRef] [PubMed]

76. Cassini, A.; Hogberg, L.D.; Plachouras, D.; Quattrocchi, A.; Hoxha, A.; Simonsen, G.S.; Colomb-Cotinat, M.; Kretzschmar, M.E.; Devleesschauwer, B.; Cecchini, M.; et al. Attributable deaths and disability-adjusted life-years caused by infections with antibiotic-resistant bacteria in the EU and the European Economic Area in 2015: A population-level modelling analysis. Lancet Infect. Dis. 2018, in press. [CrossRef]

77. Kampf, G. Antiseptic stewardship for alcohol-based hand rubs. In Antiseptic Stewardship: Biocide Resistance and Clinical Implications; Kampf, G., Ed.; Springer International Publishing: Cham, Switzerland, 2018; pp. 643-650.

78. Kampf, G.; Kramer, A.; Suchomel, M. Lack of sustained efficacy for alcohol-based surgical hand rubs containing "residual active ingredients" according to en 12791. J. Hosp. Infect. 2017, 95, 163-168. [CrossRef] [PubMed]

79. WHO. Global Guidelines for the Prevention of Surgical Site Infections; WHO: Geneva, Switzerland, 2016.

80. KRINKO am Robert Koch Institut. Händehygiene in Einrichtungen des Gesundheitswesens. Bundesgesundheitsbl 2016, 59, 1189-1220. [CrossRef] [PubMed]

81. Kampf, G. Antiseptic stewardship for skin antiseptics. In Antiseptic Stewardship: Biocide Resistance and Clinical Implications; Kampf, G., Ed.; Springer International Publishing: Cham, Switzerland, 2018; pp. 651-660.

82. Harnoss, J.C.; Assadian, O.; Kramer, A.; Probst, P.; Muller-Lantzsch, C.; Scheerer, L.; Bruckner, T.; Diener, M.K.; Buchler, M.W.; Ulrich, A.B. Comparison of chlorhexidine-isopropanol with isopropanol skin antisepsis for prevention of surgical-site infection after abdominal surgery. Br. J. Surg. 2018, 105, 893-899. [CrossRef]

83. Darouiche, R.O.; Wall, M.J.; Itani, K.M.; Otterson, M.F.; Webb, A.L.; Carrick, M.M.; Miller, H.J.; Awad, S.S.; Crosby, C.T.; Mosier, M.C.; et al. Chlorhexidine-alcohol versus povidone-iodine for surgical-site antisepsis. N. Engl. J. Med. 2010, 362, 18-26. [CrossRef]

84. Tuuli, M.G.; Liu, J.; Stout, M.J.; Martin, S.; Cahill, A.G.; Odibo, A.O.; Colditz, G.A.; Macones, G.A. A randomized trial comparing skin antiseptic agents at cesarean delivery. N. Engl. J. Med. 2016, 374, 647-655. [CrossRef]

85. Chaiyakunapruk, N.; Veenstra, D.L.; Lipsky, B.A.; Saint, S. Chlorhexidine compared with povidone-iodine solution for vascular catheter-site care: A meta-analysis. Ann. Intern. Med. 2002, 136, 792-801. [CrossRef] 
86. Mimoz, O.; Lucet, J.C.; Kerforne, T.; Pascal, J.; Souweine, B.; Goudet, V.; Mercat, A.; Bouadma, L.; Lasocki, S.; Alfandari, S.; et al. Skin antisepsis with chlorhexidine-alcohol versus povidone iodine-alcohol, with and without skin scrubbing, for prevention of intravascular-catheter-related infection (clean): An open-label, multicentre, randomised, controlled, two-by-two factorial trial. Lancet 2015, 386, 2069-2077. [CrossRef]

87. Dettenkofer, M.; Wilson, C.; Gratwohl, A.; Schmoor, C.; Bertz, H.; Frei, R.; Heim, D.; Luft, D.; Schulz, S.; Widmer, A.F. Skin disinfection with octenidine dihydrochloride for central venous catheter site care: A double-blind, randomized, controlled trial. Clin. Microbiol. Infect. 2010, 16, 600-606. [CrossRef] [PubMed]

88. Lutz, J.T.; Diener, I.V.; Freiberg, K.; Zillmann, R.; Shah-Hosseini, K.; Seifert, H.; Berger-Schreck, B.; Wisplinghoff, H. Efficacy of two antiseptic regimens on skin colonization of insertion sites for two different catheter types: A randomized, clinical trial. Infection 2016, 44, 707-712. [CrossRef] [PubMed]

89. Bourke, P.; Ziuzina, D.; Han, L.; Cullen, P.J.; Gilmore, B.F. Microbiological interactions with cold plasma. J. Appl. Microbiol. 2017, 123, 308-324. [CrossRef] [PubMed]

(c) 2018 by the author. Licensee MDPI, Basel, Switzerland. This article is an open access article distributed under the terms and conditions of the Creative Commons Attribution (CC BY) license (http:/ / creativecommons.org/licenses/by/4.0/). 